\title{
Uniformisations partielles et critères à la Hurewicz dans le plan.
}

\author{
Dominique LECOMTE
}

Trans. Amer. Math. Soc. 347, 11 (1995), 4433-4460

\begin{abstract}
Résumé. On donne des caractérisations des boréliens potentiellement d'une classe de Wadge donnée, parmi les boréliens à coupes verticales dénombrables d'un produit de deux espaces polonais. Pour ce faire, on utilise des résultats d'uniformisation partielle.
\end{abstract}

Cet article fait suite à l'étude des classes de Wadge potentielles commencée dans [Le1]. On démontre entre autres les résultats annoncés dans [Le2]. Je renvoie le lecteur à [Ku] pour ce qui est des notions de base en topologie, et à [W] pour ce qui concerne les classes de Wadge. On utilisera les notations et notions standard de la théorie descriptive des ensembles, ainsi que des notions de théorie descriptive effective, qui peuvent être trouvées dans [Mo]. Rappelons les définitions de base :

Définitions. (a) Soit $\Gamma$ une classe de parties d'espaces polonais de dimension 0 . On dit que $\Gamma$ est une classe de $W$ adge de boréliens s'il existe un espace polonais $P_{0}$ de dimension 0 , et un borélien $A_{0}$ de $P_{0}$ tels que pour tout espace polonais $P$ de dimension 0 et pour toute partie $A$ de $P$, A est dans $\Gamma$ si et seulement s'il existe une fonction continue $f$ de $P$ dans $P_{0}$ telle que $A=f^{-1}\left(A_{0}\right)$.

(b) Soient $X$ et $Y$ des espaces polonais, et $A$ un borélien de $X \times Y$. Si $\Gamma$ est une classe de Wadge, on dira que A est potentiellement dans $\Gamma$ (ce qu'on notera $A \in \operatorname{pot}(\Gamma))$ s'il existe des topologies polonaises de dimension $0, \sigma($ sur $X)$ et $\tau$ (sur $Y)$, plus fines que les topologies initiales, telles que $A$, considéré comme partie de $(X, \sigma) \times(Y, \tau)$, soit dans $\Gamma$.

La motivation pour l'étude de ces classes de Wadge potentielles trouve son origine dans l'étude des relations d'équivalence boréliennes (ou plus généralement des structures boréliennes à plusieurs variables). Par exemple, dans [HKL], on étudie le pré-ordre qui suit. Si $E$ (resp. $E^{\prime}$ ) est une relation d'équivalence borélienne sur l'espace polonais $X$ (resp. $X^{\prime}$ ), on pose

$$
\left.E \leq E^{\prime} \Leftrightarrow \text { [il existe } f \text { borélienne de } X \text { dans } X^{\prime} \text { telle que } x E y \Leftrightarrow f(x) E^{\prime} f(y)\right]
$$

En d'autres termes, l'application $f$ définit par passage au quotient une injection de $X / E$ dans $X^{\prime} / E^{\prime}$, ce de manière borélienne. La relation $E \leq E^{\prime}$ peut s'écrire : $E=(f \times f)^{-1}\left(E^{\prime}\right)$, avec $f$ borélienne ; or si $E^{\prime}$ est de classe de Wadge $\Gamma$ (ou même de classe de Wadge potentielle $\Gamma$ ), il est facile de vérifier que $E$ est de classe de Wadge potentielle $\Gamma$ (alors que $E$ n'est pas de classe $\Gamma$ en général). La classe de Wadge potentielle est donc un invariant naturel du pré-ordre $\leq$. Bien que cet invariant soit en général grossier, il fournit des informations sur $\leq$; par exemple, dans [Lo1], A. Louveau montre que la classe des relations d'équivalence $\Sigma_{\xi}^{0}$ n'est pas co-finale dans les relations d'équivalence boréliennes pour le pré-ordre $\leq$, en utilisant la notion de classe de Wadge potentielle. 
Il en déduit une démonstration simple de la non-existence d'une relation d'équivalence borélienne maximum pour $\leq$. Ce résultat avait par ailleurs été démontré antérieurement par $\mathrm{H}$. Friedman et $\mathrm{L}$. Stanley, en utilisant les travaux de H. Friedman sur la diagonalisation borélienne.

L'un des principaux résultats concernant les classes de Wadge de boréliens est l'existence de "tests d'Hurewicz", dont le principe est : un borélien n'est pas d'une classe de Wadge donnée si et seulement s'il est au moins aussi compliqué qu'un exemple type n'étant pas de cette classe. Hurewicz a démontré l'existence du test pour la classe $G_{\delta}$. Le résultat précis est le

Théorème 2.10 Soit $X$ un espace polonais, et $A$ un borélien de $X$. Alors $A$ n'est pas $G_{\delta}$ si et seulement s'il existe $E$ dénombrable sans point isolé tel que $\bar{E} \backslash E \approx \omega^{\omega}$ et $E=A \cap \bar{E}$.

Après les travaux de nombreux auteurs, parmi lesquels J. R. Steel (voir [S]), ainsi que A. Louveau et J. Saint Raymond, l'existence de tests a été établie pour toutes les classes de Wadge ; dans [Lo-SR], il est démontré :

Théorème. Si $\xi$ est un ordinal dénombrable non nul, il existe un compact $P_{\xi}$ de dimension 0 et un vrai $\Sigma_{\xi}^{0}$ de $P_{\xi}$, $A_{\xi}$, tels que si $A$ est un borélien de l'espace polonais $X$, on ait : A n'est pas $\Pi_{\xi}^{0}$ de $X$ si et seulement s'il existe $f: P_{\xi} \rightarrow X$ injective continue telle que $A_{\xi}=f^{-1}(A)$.

L'ensemble $A_{\xi}$ est dit “test d'Hurewicz". Un des objectifs de cet article est d'étudier la possibilité d'obtenir des résultats similaires, pour les classes de Wadge potentielles. Dans [Le1], il est démontré un lemme qui suggère l'intérêt qu'on pourrait avoir à étudier les problèmes d'uniformisation partielle, en vue de caractériser les ensembles potentiellement fermés. Le test usuel pour savoir si un ensemble $A$ est fermé est de prendre une suite convergente de points de $A$ et de regarder si la limite est dans $A$. Un tel test ne peut pas convenir pour caractériser les ensembles potentiellement fermés, puisqu' un singleton peut être rendu ouvert-fermé tout en gardant des topologies polonaises. Cependant, on remarque que lorsqu'on raffine la topologie d'un espace polonais, tout en gardant une topologie polonaise, les deux topologies coïncident sur un $G_{\delta}$ dense pour la topologie initiale. D'où l'idée de remplacer les points par des graphes de fonctions continues et ouvertes, objets qui rencontrent tout produit de deux $G_{\delta}$ denses si les domaines et images des fonctions sont assez "gros".

Lemme. Soient $X$ et $Y$ des espaces polonais, $\left(C_{n}\right)$ (resp. $\left.\left(D_{n}\right)\right)$ des suites d'ouverts non vides de $X$ (resp. $Y$ ), $f_{n}: C_{n} \rightarrow D_{n}$ continues et ouvertes, $B:=\bigcup_{n \in \omega \backslash\{0\}} \operatorname{Gr}\left(f_{n}\right)$, et $A$ un borélien de $X \times Y$ contenant B; si $\bar{B} \backslash A$ contient $G r\left(f_{0}\right)$, alors A est non-pot $\left(\boldsymbol{\Pi}_{1}^{0}\right)$.

La question est de savoir s'il y a une réciproque. Nous allons voir que c'est en partie le cas : cette réciproque a lieu, modulo un changement de topologies, si $A$ est à la fois $\operatorname{pot}\left(\boldsymbol{\Sigma}_{3}^{0}\right)$ et $\operatorname{pot}\left(\boldsymbol{\Pi}_{3}^{0}\right)$, à ceci près qu'on ne peut pas imposer le sens dans lequel se trouvent les graphes. Ceci signifie que les fonctions partielles peuvent être définies sur une partie de $X$ et arriver dans $Y$, ou être définies sur une partie de $Y$ et arriver dans $X$. Le résultat précis est un cas particulier du théorème 2.3 ; si $f_{n}$ est une fonction partielle de $X$ dans $Y$ ou de $Y$ dans $X$, on notera $G_{n}$ la partie de $X \times Y$ égale au graphe de $f_{n}$ si $f_{n}$ va de $X$ dans $Y$, et égale à $\left\{(x, y) \in X \times Y / x=f_{n}(y)\right\}$ sinon. 
Théorème *. Soient $X$ et $Y$ des espaces polonais, et $A$ un borélien $\operatorname{pot}\left(\boldsymbol{\Sigma}_{3}^{0}\right) \cap \operatorname{pot}\left(\boldsymbol{\Pi}_{3}^{0}\right)$ de $X \times Y$. A est non-pot $\left(\Pi_{1}^{0}\right)$ si et seulement s'il existe des espaces polonais $Z$ et $T$ parfaits de dimension 0 , des ouverts-fermés non vides $A_{n}$ et $B_{n}$ (l'un dans $Z$ et l'autre dans $T$ ), des surjections continues ouvertes $f_{n}: A_{n} \rightarrow B_{n}$, et des injections continues $u$ et $v$ tels que $\bigcup_{n \in \omega \backslash\{0\}} G_{n} \subseteq(u \times v)^{-1}(A)$, $G_{0} \subseteq(u \times v)^{-1}(\check{A})$, et $G_{0}=\overline{\bigcup_{n \in \omega \backslash\{0\}} G_{n}} \backslash\left(\bigcup_{n \in \omega \backslash\{0\}} G_{n}\right)$.

Le théorème 2.3 étend ce résultat à la caractérisation des boréliens potentiellement différence transfinie d'ouverts, toujours parmi les boréliens $\operatorname{pot}\left(\Sigma_{3}^{0}\right) \cap \operatorname{pot}\left(\boldsymbol{\Pi}_{3}^{0}\right)$. On ne se ramène donc pas à un exemple type, comme dans le théorème de A. Louveau et J. Saint Raymond, mais à une situation type. Pour démontrer le théorème 2.3, l'outil essentiel est le

Théorème 1.13 Soient $X$ et $Y$ des espaces polonais parfaits de dimension $0, A$ un $G_{\delta}$ l.p.o. non vide de $X \times Y$. Alors il existe des ensembles presque-ouverts non vides $F$ et $G$, l'un contenu dans $X$ et l'autre dans $Y$, et une surjection continue ouverte de $F$ sur $G$ dont le graphe est contenu dans $A$ ou dans $\{(y, x) \in Y \times X /(x, y) \in A\}$ selon le cas.

Pour le comprendre voici les

Définitions 1.2 (a) Un $G_{\delta}$ d'un espace topologique est dit presque-ouvert (ou p.o.) s'il est contenu dans l'intérieur de son adhérence (ce qui revient à dire qu'il est dense dans un ouvert).

(b) Si $X$ et $Y$ sont des espaces topologiques, une partie A de $X \times Y$ sera dite localement à projections ouvertes (ou l.p.o.) si pour tout ouvert $U$ de $X \times Y$, les projections de $A \cap U$ sont ouvertes.

Les ensembles 1.p.o. se rencontrent par exemple dans la situation suivante : $A$ est $\Sigma_{1}^{1}$ dans un produit de deux espaces polonais récursivement présentés. Si on munit ces deux espaces de leur topologie de Gandy-Harrington (celle engendrée par les $\Sigma_{1}^{1}$ ), $A$ devient 1.p.o. dans le nouveau produit. C'est essentiellement dans cette situation qu'on utilisera cette notion, au cours de la section 2.

On étudie donc dans un premier temps, et plus largement que nécessaire pour la seule étude des classes de Wadge potentielles, les problèmes d'uniformisation partielle, sur des ensembles "gros" au sens de la catégorie, en essayant d'obtenir l'image de la fonction "grosse" également. On obtient essentiellement des résultats pour les $G_{\delta}$. Il est à noter que malgré des hypothèses symétriques, la conclusion du théorème 1.13 ne l'est pas.

Dans [O], il est démontré, sous l'hypothèse du continu, l'existence d'une bijection $\Phi$ de $[0,1]$ sur lui même échangeant ensembles maigres et ensembles de mesure de Lebesgue nulle. En étudiant un exemple où on trouve un graphe dans un sens et pas dans l'autre, dans le théorème 1.13, on montre qu'une telle application $\Phi$ ne peut pas avoir de propriété de mesurabilité projective (corollaire 1.8).

Une autre façon de formuler le théorème * est (voir [Le2]) le

Théorème. Soient $X$ et $Y$ des espaces polonais, A un borélien de $X \times Y$ à coupes verticales dénombrables. Alors A est non-pot $\left(\Pi_{1}^{0}\right)$ si et seulement s'il existe des espaces polonais $Z$ et $T$ parfaits de dimension 0 non vides, des fonctions continues $u$ et $v$, des ouverts denses $\left(A_{n}\right)$ de $Z$, des applications continues ouvertes $f_{n}$ de $A_{n}$ dans $T$, tels que pour tout $x$ dans $\bigcap_{n \in \omega} A_{n},\left(f_{n}(x)\right)_{n>0}$ converge simplement vers $f_{0}(x), G r\left(g_{0}\right) \subseteq(u \times v)^{-1}(\check{A})$ et $\bigcup_{n>0} G r\left(g_{n}\right) \subseteq(u \times v)^{-1}(A)$. 
En d'autres termes, en chaque point du $G_{\delta}$ dense $\bigcap_{n \in \omega} A_{n}$, on retrouve sur la fibre le test usuel pour les fermés. On a un phénomène analogue pour les $G_{\delta}$ :

Théorème 2.11 Soient $X$ et $Y$ des espaces polonais, $A$ un borélien de $X \times Y$ à coupes verticales dénombrables. Alors $A$ est non-pot $\left(\boldsymbol{\Pi}_{2}^{0}\right)$ si et seulement s'il existe des espaces polonais $Z$ et $T$ parfaits de dimension 0 non-vides, des injections continues u et $v$, des ouverts denses $\left(A_{n}\right)$ de $Z$, des applications continues et ouvertes $f_{n}$ de $A_{n}$ dans $T$, tels que pour tout $x$ dans $\bigcap_{n \in \omega} A_{n}$, l'ensemble $E_{x}:=\left\{f_{n}(x) / n \in \omega\right\}$ soit sans point isolé, $\overline{E_{x}} \backslash E_{x} \approx \omega^{\omega}$, et $E_{x}=(u \times v)^{-1}(A)_{x} \cap \overline{E_{x}}$.

\section{Uniformisation partielle des $G_{\delta}$.}

Les problèmes d'uniformisation partielle ont déjà été étudiés dans [GM], où au lieu de considérer la catégorie, il est question d'ensembles de mesure 1 sur chacun des facteurs. Les résultats qu'on obtient sont également à rapprocher de résultats obtenus par G. Debs et J. Saint Raymond, où il est question de fonctions totales et injectives, avec des hypothèses de compacité sur chacun des facteurs (cf [D-SR]). Plus précisément, il est démontré dans [GM] le résultat suivant :

Théorème 1.1 Soient $X$ et $Y$ des espaces polonais, $\lambda($ resp. $\mu)$ une mesure de probabilité sur $X$ (resp. $Y$ ), et $A$ un borélien de $X \times Y$ ayant ses coupes horizontales (resp. verticales) non dénombrables $\mu$-presque partout (resp. $\lambda$-presque partout). Alors il existe un borélien $F$ de $X$ (resp. $G$ de $Y$ ) tels que $\lambda(F)=\mu(G)=1$, et un isomorphisme borélien de $F$ sur $G$ dont le graphe est contenu dans $A$.

On peut se demander si on a un résultat analogue en remplaçant "ensemble de mesure 0" par "ensemble maigre". On va voir que non. Pour ce faire on montre un lemme que nous réutiliserons.

Définitions 1.2 (a) Un $G_{\delta}$ d'un espace topologique est dit presque-ouvert (ou p.o.) s'il est contenu dans l'intérieur de sn adhérence (ce qui revient à dire qu'il est dense dans un ouvert).

(b) Si $X$ et $Y$ sont des espaces topologiques, une partie A de $X \times Y$ sera dite localement à projections ouvertes (ou l.p.o.) si pour tout ouvert $U$ de $X \times Y$, les projections de $A \cap U$ sont ouvertes.

Lemme 1.3 Il existe un espace $X$ polonais parfait de dimension 0 , et un fermé $A$ l.p.o. à coupes parfaites non vides de $X^{2}$, tels que si $F$ et $G$ sont presque-ouverts non vides dans $X$ et $f: F \rightarrow G$ surjective continue ouverte, $\operatorname{Gr}(f) \nsubseteq A$.

Démonstration. Soient $X=\omega^{\omega}, \phi$ un homéomorphisme de $X$ sur l'ensemble $P_{\infty}$ des suites de 0 et de 1 ayant une infinité de 1 , et $\psi$ un homéomorphisme de $X$ sur l'ensemble $\mathcal{K}_{P}\left(2^{\omega}\right)$ des compacts parfaits non vides de $2^{\omega} ; \psi$ existe car cet ensemble ne contient pas les compacts finis, est dense et est $G_{\delta}$ de $\mathcal{K}\left(2^{\omega}\right) \backslash\{\emptyset\}$ : en effet, si on désigne par $N\left(n, 2^{\omega}\right)$ le $n^{\text {ième }}$ ouvert-fermé de base de $2^{\omega}$, on a $K$ est parfait $\Leftrightarrow\left\{\begin{array}{l}\forall m \in \omega\left[\left(K \cap N\left(m, 2^{\omega}\right)=\emptyset\right) \text { ou }\left(\exists(p, q) \in \omega^{2} N\left(p, 2^{\omega}\right) \cap N\left(q, 2^{\omega}\right)=\emptyset \text { et }\right.\right. \\ \left.\left.N\left(p, 2^{\omega}\right) \cup N\left(q, 2^{\omega}\right) \subseteq N\left(m, 2^{\omega}\right) \text { et } N\left(p, 2^{\omega}\right) \cap K \neq \emptyset \text { et } N\left(q, 2^{\omega}\right) \cap K \neq \emptyset\right)\right] .\end{array}\right.$ 
Posons $A:=(\phi \times \psi)^{-1}\left(A^{\prime}\right)$, où $A^{\prime}:=\left\{(x, K) \in P_{\infty} \times \mathcal{K}_{P}\left(2^{\omega}\right) / x \in K\right\}$. Il suffit de montrer les propriétés pour $A^{\prime}$. Il est fermé :

$$
x \notin K \Leftrightarrow \exists n \in \omega \exists U \in \Sigma_{1}^{0}\left\lceil 2^{\omega} N\left(n, 2^{\omega}\right) \cap U=\emptyset \text { et } x \in N\left(n, 2^{\omega}\right) \text { et } K \subseteq U .\right.
$$

Il est clairement à coupes parfaites non vides, et il est 1.p.o., car si $N_{s}:=\left\{\alpha \in 2^{\omega} / s \prec \alpha\right\}$ et

$$
W_{U, V_{0}, \ldots, V_{n}}:=\left\{K \in \mathcal{K}_{P}\left(2^{\omega}\right) / K \subseteq U \text { et } \forall i \leq n V_{i} \cap K \neq \emptyset\right\}
$$

est l'ouvert-fermé de base de $\mathcal{K}_{P}\left(2^{\omega}\right)$, on a

$$
\begin{gathered}
\Pi_{1}\left[A^{\prime} \cap\left(N_{s} \times W_{U, V_{0}, \ldots, V_{n}}\right)\right]=\emptyset \text { ou } N_{s} \cap U \cap P_{\infty}, \text { et } \\
\Pi_{2}\left[A^{\prime} \cap\left(N_{s} \times W_{U, V_{0}, \ldots, V_{n}}\right)\right]=\emptyset \text { ou } W_{U, V_{0}, \ldots, V_{n}, N_{s}} \cap \mathcal{K}_{P}\left(2^{\omega}\right) .
\end{gathered}
$$

Raisonnons par l'absurde : il existe $f: F \rightarrow G$ surjective continue ouverte dont le graphe est contenu dans $A^{\prime}$, et des ouverts non vides $\mathcal{U}$ et $\mathcal{V}$ tels que $F$ (resp. $G$ ) soit $G_{\delta}$ dense de $\mathcal{U}$ (resp. $\mathcal{V}$ ).

L'ouvert $\mathcal{V}$ étant non vide contient un ouvert-fermé de base $W_{U, V_{0}, \ldots, V_{n}}$, avec $U, V_{0}, \ldots, V_{n}$ ouverts-fermés tels que les $U \cap V_{i}$ soient non vides. De sorte qu'on peut supposer, quitte à se restreindre à son image réciproque, que $\mathcal{V}=W_{U, V_{0}, \ldots, V_{n}}$.

Soit $\left(S_{j}\right)_{j<n+3}$ une partition de $U$ en ouverts-fermés telle que $\forall j<n+3, \forall i \leq n, S_{j} \cap V_{i} \neq \emptyset$. Posons, si $I \in \mathcal{P}(n+3) \backslash\{\emptyset\}$,

$$
O_{I}:=\left\{K \in W_{U, V_{0}, \ldots, V_{n}} / I=\left\{j<n+3 / K \cap S_{j} \neq \emptyset\right\}\right\}
$$

Alors $\left(O_{I}\right)$ est une partition en ouverts-fermés non vides de $W_{U, V_{0}, \ldots, V_{n}}$, donc $\left(f^{-1}\left(O_{I}\right)\right)$ est une partition en ouverts-fermés non vides de $F$; par propriété de réduction, on trouve une suite d'ouverts non vides de $\mathcal{U}$ deux à deux disjoints, $\left(W_{I}\right)$, telle que $f^{-1}\left(O_{I}\right)=F \cap W_{I}$.

Alors si $j<n+3, W_{\{j\}} \subseteq S_{j}$, sinon on trouve $x$ dans $F \cap W_{\{j\}} \backslash S_{j}$, et $x \in f(x) \in O_{\{j\}}$, donc $f(x) \subseteq S_{j}$, une contradiction.

Maintenant si $I \subseteq n+3$ est de cardinal 2, il existe $i \leq n$ tel que $V_{i} \cap \bigcup_{j \in I} S_{j} \subseteq \overline{W_{I}}$. En effet, par ce qui précède, si $j \in I, S_{j} \nsubseteq \overline{W_{I}}$ sinon $W_{\{j\}} \cap W_{I} \neq \emptyset$. Donc si pour tout $i \leq n$ il existe $j$ dans $I$ tel que $V_{i} \cap S_{j} \nsubseteq \overline{W_{I}}$, l'ensemble $\left\{K \in O_{I} / K \subseteq \overline{W_{I}}\right\}$ est ouvert non vide de $\bar{G}$, donc rencontre $G$ en $K=f(x)$ et $x \in F \cap W_{I}$, donc $K \cap W_{I}$ est non vide, une contradiction.

En particulier, si $1 \leq j \leq n+2$, il existe $i_{j} \leq n$ tel que $V_{i_{j}} \cap\left(S_{0} \cup S_{j}\right) \subseteq \overline{W_{\{0, j\}}}$. Soient $j \neq j^{\prime}$ tels que $i_{j}=i_{j^{\prime}}$; alors $V_{i_{j}} \cap S_{0} \subseteq \overline{W_{\{0, j\}}} \cap \overline{W_{\left\{0, j^{\prime}\right\}}}$, ce qui contredit la disjonction de $W_{\{0, j\}}$ et $W_{\left\{0, j^{\prime}\right\}}$.

Théorème 1.4 Soient $X:=\mathcal{K}\left(2^{\omega}\right) \backslash\{\emptyset\}, Y:=2^{\omega}$, et $A:=\{(K, x) \in X \times Y / x \in K\}$; alors $A$ est un fermé à coupes horizontales non dénombrables, à coupes verticales non dénombrables sur un ensemble co-maigre de $X$, mais si $F$ (resp. $G$ ) est un borélien co-maigre de $X$ (resp. $Y$ ), il n'existe aucun isomorphisme borélien de $F$ sur $G$ dont le graphe soit contenu dans $A$. 
Démonstration. Comme on l'a vu dans la preuve du lemme précédent, $A$ est fermé dans $X \times Y$, et l'ensemble des compacts parfaits non vides est $G_{\delta}$ dense de $X$.

L'ensemble des compacts non dénombrables est donc co-maigre dans $X$, et les coupes verticales de $A$ sont donc non dénombrables, sauf sur un maigre.

Raisonnons par l'absurde : il existe des ensembles $F$ et $G$, ainsi qu'un isomorphisme borélien $f$ comme dans l'énoncé.

Alors il existe un $G_{\delta}$ dense $F^{\prime}$ (resp. $\left.G^{\prime}\right)$ de $X$ (resp. $Y$ ) tels que $F^{\prime} \subseteq \mathcal{K}_{P}\left(2^{\omega}\right) \cap F, G^{\prime} \subseteq G \cap P_{\infty}$, et que $f: F^{\prime} \rightarrow G^{\prime}$ soit un homéomorphisme.

En effet, on remarque que si $U$ est un ouvert dense de $Y$, alors $\{K \in X / K \subseteq U\}$ est un ouvert dense de $X$; par suite, si $M$ est maigre relativement à $G$, on a l'inclusion

$$
f^{-1}(M) \subseteq \Pi_{X}[(X \times M) \cap A]=\{K \in X / K \cap M \neq \emptyset\} .
$$

Donc $f^{-1}(M)$ est maigre relativement à $X$, et aussi relativement à $F$ qui est co-maigre dans $X$.

Soit $G_{1}$ un $G_{\delta}$ dense de $Y$, contenu dans $G \cap P_{\infty}$, sur lequel $f^{-1}$ est continue ; ce qui précède montre que $f^{-1}\left(G_{1}\right)$ est co-maigre dans $X$.

Si $\left(U_{n}\right)$ est une base de la topologie de $G_{1}, f^{-1}\left(U_{n}\right)$ est borélien de $X$, donc égal à un ouvert $V_{n}$ modulo un maigre $M_{n}$; choisissons pour $F^{\prime}$ un $G_{\delta}$ dense de $X$ contenu dans

$$
\mathcal{K}_{P}\left(2^{\omega}\right) \cap F \cap f^{-1}\left(G_{1}\right) \backslash\left(\bigcup_{n \in \omega} M_{n}\right) .
$$

Il reste à poser $G^{\prime}:=f^{\prime \prime} F^{\prime}$.

En effet, on a $f^{-1}\left(U_{n} \cap G^{\prime}\right)=F^{\prime} \cap f^{-1}\left(U_{n}\right)=F^{\prime} \cap V_{n} ; G^{\prime}=\left(f^{-1}\right)^{-1}\left(F^{\prime}\right) \cap G_{1}$ est $G_{\delta}$. Et si $G^{\prime}$ n'était pas dense dans $Y$, on trouverait un ouvert non vide $U$ de $Y$ disjoint de $G^{\prime}$; mais $\{K \in X / K \subseteq U\}$ serait un ouvert non vide, et rencontrerait donc $F^{\prime}$ en un point $K$ qui vérifierait $f(K) \in K \subseteq U$ et aussi $f(K) \in G^{\prime}$, ce qui est la contradiction cherchée.

Mais ceci contredit la preuve du lemme 1.3.

En analysant les raisons de ce résultat négatif, nous allons maintenant montrer que l'application transformant ensembles de mesure 0 en ensembles maigres n'a pas de propriétés de mesurabilité projective, sous hypothèse de détermination des jeux projectifs.

Pour démontrer le théorème 1.1, les auteurs montrent le lemme suivant :

Lemme 1.5 Soient $X$ un espace polonais, $\mu$ une mesure de probabilité sur $X$, et $A$ un borélien de $X \times X$ ayant ses coupes horizontales non dénombrables $\mu$-presque partout. Alors il existe un $K_{\sigma}$ de $X, F$, et une application borélienne $f: F \rightarrow X$ tels que $G r(f) \subseteq A$ et

$$
\mu\left(\left\{y \in X / f^{-1}(\{y\}) \text { est non dénombrable }\right\}\right)=1 .
$$


Corollaire 1.6 Soient $B:=[0,1], \lambda$ la mesure de Lebesgue sur $B, A$ un borélien de $B \times B$ tel que $\lambda\left(\left\{y \in B / A^{y}\right.\right.$ est non dénombrable $\left.\}\right)=1$; alors il existe des boréliens disjoints de $B, B_{0}$ et $B_{1}$, tels que $\lambda\left(\left\{y \in B / B_{i} \cap A^{y}\right.\right.$ est non dénombrable $\left.\}\right)=1$.

Démonstration. Soient $F$ et $f$ fournis par le lemme 1.5 , et $G_{y}$ une copie de $2^{\omega}$ contenue dans $f^{-1}(\{y\})$, si ce borélien est non dénombrable. Si $x \in G_{y} \backslash\left\{\min G_{y}, \max G_{y}\right\}$, les ouverts $\left[0, x\left[\cap G_{y}\right.\right.$ et $] x, 1] \cap G_{y}$ sont non vides, donc non dénombrables ; posons donc

$$
E(y, x) \Leftrightarrow x \in F \text { et } f(x)=y \text { et }\left[0, x\left[\cap f^{-1}(\{y\}),\right] x, 1\right] \cap f^{-1}(\{y\}) \text { sont non dénombrables. }
$$

Alors $E$ est analytique dans $B \times B$, donc par le théorème de von Neumann on trouve une application $g: B \rightarrow B$ Baire-mesurable telle que si $f^{-1}(\{y\})$ est non dénombrable, $g(y) \in F, f(g(y))=y$, et $\left[0, g(y)\left[\cap f^{-1}(\{y\}),\right] g(y), 1\right] \cap f^{-1}(\{y\})$ sont non dénombrables.

Soit alors $G$ un borélien de $B$ tel que $\lambda(G)=1$, la restriction de $g$ à $G$ soit borélienne, et contenu dans $\left\{y \in B / f^{-1}(\{y\})\right.$ est non dénombrable $\}$. Il reste à poser

$$
B_{0}:=\{x \in F / f(x) \in G \text { et } x<g(f(x))\}, B_{1}:=\{x \in F / f(x) \in G \text { et } x>g(f(x))\} .
$$

En effet, si $y \in G,\left[0, g(y)\left[\cap f^{-1}(\{y\}) \subseteq B_{0} \cap A^{y}\right.\right.$, donc $B_{0} \cap A^{y}$ est non dénombrable, de même que $B_{1} \cap A^{y}$.

Lemme 1.7 Il existe un $G_{\delta}$ de $B \times B, A$, où $B:=[0,1]$, tel que $\left\{y \in B / A^{y}\right.$ est non dénombrable $\}$ soit co-maigre, alors que pour tout couple $\left(B_{0}, B_{1}\right)$ de boréliens disjoints de $B$,

$$
\left\{y \in B / B_{i} \cap A^{y} \text { est non dénombrable }\right\}
$$

ne sont pas tous deux co-maigres.

Démonstration. $[0,1] \backslash \mathbb{Q}$ est homéomorphe à $P_{\infty}$, qui est co-dénombrable dans l'espace parfait $2^{\omega}$. Il suffit donc de trouver $A$ dans $2^{\omega} \times 2^{\omega}$, ayant les propriétés du lemme. D'autre part, $\mathcal{K}\left(2^{\omega}\right) \backslash\{\emptyset\}$ est un espace compact métrisable parfait de dimension 0 et non vide, donc est homéomorphe à $2^{\omega}$; il suffit donc de trouver $A$ dans $2^{\omega} \times \mathcal{K}\left(2^{\omega}\right) \backslash\{\emptyset\}$. Reprenons l'exemple de 1.4 :

$$
A=\left\{(x, K) \in 2^{\omega} \times \mathcal{K}\left(2^{\omega}\right) \backslash\{\emptyset\} / x \in K\right\} .
$$

Raisonnons par l'absurde : $B_{0}$ et $B_{1}$ existent. Par la preuve de 1.4, ces deux ensembles sont nécessairement non maigres ; on trouve donc des ouverts disjoints et non vides de $2^{\omega}, U_{0}$ et $U_{1}$, tels que $B_{0} \Delta U_{0}$ et $\check{B}_{0} \Delta U_{1}$ soient maigres.

Comme on l'a vu dans la preuve du théorème 1.4, $\left\{K \in \mathcal{K}\left(2^{\omega}\right) \backslash\{\emptyset\} / K \cap\left(B_{0} \Delta U_{0}\right)=\emptyset\right\}$ est co-maigre, ainsi que $\left\{K \in \mathcal{K}\left(2^{\omega}\right) \backslash\{\emptyset\} / B_{0} \cap K\right.$ est non dénombrable $\}$, donc que l'ensemble $\left\{K \in \mathcal{K}\left(2^{\omega}\right) \backslash\{\emptyset\} / K \cap U_{0} \neq \emptyset\right\}$; ce dernier rencontre donc l'ouvert non vide

$$
\left\{K \in \mathcal{K}\left(2^{\omega}\right) \backslash\{\emptyset\} / K \subseteq U_{1}\right\}
$$

ce qui contredit la disjonction de $U_{0}$ et $U_{1}$.

Il résulte immédiatement du théorème 19.6 de [O] que sous l'hypothèse du continu, il existe une bijection idempotente $\Phi$ de $B:=[0,1]$ sur lui-même telle que $E$ est maigre si et seulement si $\lambda\left(\Phi^{-1}(E)\right)=0$. Ce qui précède entraîne le 
Corollaire 1.8 Une telle fonction $\Phi$ n'est pas borélienne. De plus, sous hypothèse de détermination des jeux $\boldsymbol{\Delta}_{2 n+3}^{1}$, $\Phi$ n'est pas $\boldsymbol{\Pi}_{2 n+1}^{1}$-mesurable.

Démonstration. Le premier point résulte aussitôt du corollaire 1.6 et du lemme 1.7. Si $\Phi$ était $\Pi_{2 n+1^{-}}^{1}$ mesurable, et si $A$ est le $G_{\delta}$ du lemme 1.7 , l'ensemble $A^{\prime}:=(I \times \Phi)^{-1}(A)$ serait $\Pi_{2 n+1}^{1}$, ainsi que $E:=\left\{(y, K) \in B \times \mathcal{K}(B) \backslash\{\emptyset\} / K\right.$ est parfait et $\left.K \subseteq A^{\prime y}\right\}$; par la détermination des jeux $\boldsymbol{\Delta}_{2 n}^{1}$, $E$ serait uniformisable par un graphe partiel $\Pi_{2 n+1}^{1}$, et la fonction correspondante se prolongerait en une fonction $f$ totale $\boldsymbol{\Delta}_{2 n+3}^{1}$-mesurable. Par la détermination des jeux $\boldsymbol{\Delta}_{2 n+3}^{1}, f$ serait $\lambda$-mesurable, donc il existerait un borélien $G$ de $B$ tel que $\lambda(G)=1$, la restriction de $f$ à $G$ soit borélienne, et $(y, f(y)) \in E$ si $y \in G$.

Posons $A^{\prime \prime}:=\{(x, y) \in B \times B / y \in G$ et $x \in f(y)\}$; alors $A^{\prime \prime}$ serait un borélien contenu dans $A^{\prime}$, et le corollaire 1.6 fournirait des boréliens disjoints $B_{0}$ et $B_{1}$. Les ensembles

$$
\left\{y \in B / B_{i} \cap(I \times \Phi)\left[A^{\prime \prime}\right]^{y} \text { est non dénombrable }\right\}
$$

seraient donc co-maigres, ainsi que $\left\{y \in B / B_{i} \cap A^{y}\right.$ est non dénombrable $\}$, ce qui contredirait le lemme 1.7 .

Dans [Ma], la question suivante est posée : étant donné un borélien $A$ de $[0,1] \times[0,1]$ dont toutes les coupes sont non maigres, existe-t-il un isomorphisme borélien de $[0,1]$ sur lui-même dont le graphe soit contenu dans $A$ ? La réponse est non, comme le montre le théorème 3 de [D-SR]. On a cependant le résultat suivant :

Théorème 1.9 Soient $X$ et $Y$ des espaces polonais parfaits non vides, $A \subseteq X \times Y$ ayant la propriété de Baire et ses coupes non maigres (sauf sur des ensembles maigres). Alors il existe un ensemble $K_{\sigma} \cup G_{\delta}, F$ (resp. $G$ ), co-maigre dans $X$ (resp. $Y$ ), et un isomorphisme de deuxième classe de $F$ sur $G$ dont le graphe est contenu dans $A$.

Lemme 1.10 Soient $\left(C_{n}\right),\left(D_{n}\right)$ des suites d'ouverts-fermés non vides de $\omega^{\omega}, A$ dans $\omega^{\omega} \times \omega^{\omega}$ tel que $A \Delta\left(\bigcup_{n \in \omega} C_{n} \times D_{n}\right)$ soit maigre ; alors il existe des suites $\left(K_{n}^{0}\right)$ et $\left(K_{n}^{1}\right)$ de copies de $2^{\omega}$, des suites $\left(G_{n}^{0}\right)$ et $\left(G_{n}^{1}\right)$ de $G_{\delta}$ de $\omega^{\omega}$ vérifiant

(i) $\left(K_{n}^{0} \times G_{n}^{0}\right) \cup\left(G_{n}^{1} \times K_{n}^{1}\right) \subseteq A$

(ii) $K_{n}^{0} \subseteq C_{n} \backslash\left(\bigcup_{q<n} K_{q}^{0}\right), K_{n}^{1} \subseteq D_{n} \backslash\left(\bigcup_{q<n} K_{q}^{1}\right)$

(iii) $G_{n}^{0}$ (resp. $\left.G_{n}^{1}\right)$ est dense dans $D_{n} \backslash\left(\bigcup_{q<n} D_{q} \cup \bigcup_{p \in \omega} K_{p}^{1}\right)\left(\right.$ resp. $\left.C_{n} \backslash\left(\bigcup_{q<n} C_{q} \cup \bigcup_{p \in \omega} K_{p}^{0}\right)\right)$

Démonstration. On construit d'abord la suite $\left(K_{n}^{0}\right)$ et une suite $\left(H_{n}^{0}\right)$ de $G_{\delta}$ de $\omega^{\omega}$ vérifiant

$$
K_{n}^{0} \times H_{n}^{0} \subseteq A \cap\left[C_{n} \backslash\left(\bigcup_{q<n} K_{q}^{0}\right) \times D_{n} \backslash\left(\bigcup_{q<n} D_{q}\right)\right]
$$

$H_{n}^{0}$ étant dense dans $D_{n}^{\prime}:=D_{n} \backslash\left(\bigcup_{q<n} D_{q}\right)$.

Admettons la construction effectuée pour $q<n$. Soit $C_{n}^{\prime}:=C_{n} \backslash\left(\bigcup_{q<n} K_{q}^{0}\right) ; A \cap C_{n}^{\prime} \times D_{n}^{\prime}$ est co-maigre dans $C_{n}^{\prime} \times D_{n}^{\prime}$, d'où l'existence de $K_{n}^{0}$ et $H_{n}^{0}$. 
De même, on construit des suites $\left(K_{n}^{1}\right)$ et $\left(H_{n}^{1}\right)$ vérifiant des propriétés analogues :

$$
H_{n}^{1} \times K_{n}^{1} \subseteq A \cap\left[C_{n} \backslash\left(\bigcup_{q<n} C_{q}\right) \times D_{n} \backslash\left(\bigcup_{q<n} K_{q}^{1}\right)\right],
$$

et $H_{n}^{1}$ est dense dans $C_{n} \backslash\left(\bigcup_{q<n} C_{q}\right)$. Il reste à poser $G_{n}^{0}:=H_{n}^{0} \backslash\left(\bigcup_{p \in \omega} K_{p}^{1}\right), G_{n}^{1}:=H_{n}^{1} \backslash\left(\bigcup_{p \in \omega} K_{p}^{0}\right)$. Ceci termine la preuve.

Démonstration du théorème 1.9. L'ensemble $A$ ayant la propriété de Baire, il est réunion disjointe d'un $G_{\delta}$ et d'un ensemble maigre, qui a ses coupes maigres, sauf sur des ensembles maigres ; on peut donc supposer que $A$ est $G_{\delta}$ à coupes non maigres de $\omega^{\omega} \times \omega^{\omega}, X$ et $Y$ étant parfaits non vides.

On trouve alors des suites $\left(C_{n}\right)$ et $\left(D_{n}\right)$ vérifiant les conditions du lemme 1.10, qui peut donc s'appliquer. Posons $I:=\left\{n \in \omega / G_{n}^{0} \neq \emptyset\right\}, F_{0}:=\bigcup_{n \in I} K_{n}^{0}, G_{0}:=\bigcup_{n \in I} G_{n}^{0}$. Si $n \in \omega$, $D_{n} \backslash\left(\bigcup_{q<n} D_{q} \cup \bigcup_{p \in \omega} K_{p}^{1}\right)$, donc $G_{n}^{0}$, sont denses dans $D_{n} \backslash\left(\bigcup_{q<n} D_{q}\right)$; par conséquent, $G_{0}$ est dense dans $\bigcup_{n \in \omega} D_{n}$. D'autre part, $G_{0} \in \boldsymbol{\Delta}_{1}^{0}-\mathrm{PU}\left(G_{\delta}\right)\left\lceil\left(\bigcup_{n \in \omega} D_{n}\right)\right.$, donc $G_{0}$ est $G_{\delta}$. Comme $A$ est à coupes non maigres, $\bigcup_{n \in \omega} D_{n}$ est dense dans $Y$, donc $G_{0}$ est $G_{\delta}$ dense dans $Y$.

Si $n \in I, G_{n}^{0}$ est polonais non dénombrable, donc il existe un isomorphisme de première classe $f_{n}$ de $K_{n}^{0} \operatorname{sur} G_{n}^{0}$. Alors

$$
g_{0}: \begin{cases}F_{0} & \rightarrow G_{0} \\ x & \mapsto f_{n}(x) \text { si } x \in K_{n}^{0}\end{cases}
$$

est bien définie (par (ii)), injective (par (iii)) donc bijective, de première classe par compacité de $K_{n}^{0}$, et $g_{0}^{-1}$ est aussi de première classe car $G_{n}^{0}=G_{0} \cap\left[D_{n} \backslash\left(\bigcup_{q<n} D_{q}\right)\right]$. Enfin, le graphe de $g_{0}$ est contenu dans $A$ par (i).

De même, on trouve un isomorphisme de première classe $g_{1}$, du $K_{\sigma}$ maigre de $Y F_{1}:=\bigcup_{n \in J} K_{n}^{1}$ sur le $G_{\delta}$ dense de $X, G_{1}:=\bigcup_{n \in J} G_{n}^{1}$, où $J:=\left\{n \in \omega / G_{n}^{1} \neq \emptyset\right\}$. Alors $F:=F_{0} \cup G_{1}$, $G:=F_{1} \cup G_{0}$, et

$$
f:\left\{\begin{array}{l}
F \rightarrow G \\
x \mapsto\left\{\begin{array}{l}
g_{0}(x) \text { si } x \in F_{0} \\
g_{1}^{-1}(x) \text { si } x \in G_{1}
\end{array}\right.
\end{array}\right.
$$

répondent au problème.

Venons-en à l'étude de la réciproque du lemme de l'introduction ; dans ce lemme, il est question de fonctions continues et ouvertes, donc non nécessairement injectives. Le contre-exemple du théorème 1.4 n'est donc pas un obstacle à la réciproque. De fait, l'application définie par

$$
\phi: \begin{cases}\left\{K \in \mathcal{K}\left(2^{\omega}\right) \backslash\{\emptyset\} / K \text { est parfait }\right\} & \rightarrow P_{\infty} \\ K & \mapsto \max (K)\end{cases}
$$

est surjective continue et ouverte et son graphe est contenu dans

$$
\left\{(K, x) \in \mathcal{K}\left(2^{\omega}\right) \backslash\{\emptyset\} \times 2^{\omega} / x \in K\right\} .
$$

Cependant, $\phi$ n'est injective sur aucun $G_{\delta}$ dense. Plus généralement, on a la 
Proposition 1.11 Soient $X$ un espace polonais, $Y$ un espace séparé, $f: X \rightarrow Y$ continue et ouverte. Si $f$ n'est pas injective, $f$ 'est injective sur aucun $G_{\delta}$ dense de $X$.

Démonstration. Posons $E:=\left\{\left(x, x^{\prime}\right) \in X^{2} / x \neq x^{\prime}\right.$ et $\left.f(x)=f\left(x^{\prime}\right)\right\}$. Alors $E$ est $G_{\delta}$, et l.p.o., car si $U$ et $V$ sont ouverts dans $X, \Pi[E \cap(U \times V)]=U \cap\{x \in X / f(x) \in f[V \backslash\{x\}]\}$, comme on le vérifie immédiatement. Si $f\left(x_{0}\right) \in f\left[V \backslash\left\{x_{0}\right\}\right]$, soit $x_{1} \in V \backslash\left\{x_{0}\right\}$ tel que $f\left(x_{0}\right)=f\left(x_{1}\right)$. On trouve des ouverts disjoints $W_{i}$ de $X$ tels que $x_{i} \in W_{i}, W_{1} \subseteq V$. Si $x \in f^{-1}\left(f\left[W_{1}\right]\right) \cap W_{0}$ (qui est un ouvert contenant $\left.x_{0}\right), f(x)=f(y)$, où $y \in W_{1} \subseteq V$, et $x \neq y$ car $W_{0} \cap W_{1}=\emptyset$. Si $E$ est non vide, on applique le lemme 4.4 de [Le1] pour voir que $E$ rencontre tout carré $G_{\delta}$ dense ; d'où le résultat.

Le lemme 1.3 montre qu'on ne peut pas avoir l'image "grosse" en général, dans une uniformisation du type von Neumann, même pour un fermé l.p.o. non vide d'un produit d'espaces polonais parfaits de dimension 0. Cependant, l'uniformisation souhaitée a lieu dans au moins un sens (on l'a vu avant 1.11 dans le cas particulier). Le théorème 1.13 qui suit est le résultat essentiel de ce chapitre. Il est à noter que malgré des hypothèses complètement symétriques, la conclusion ne l'est pas (cf le lemme 1.3).

Lemme 1.12 Soient $X$ et $Y$ des espaces polonais, $A$ un $G_{\delta}$ l.p.o. non vide de $X \times Y$ de projections $X$ et $Y$, et $F$ (resp. $G$ ) un $G_{\delta}$ dense de $X$ (resp. $Y$ ). Alors les projections de $A \cap(F \times G)$ sont co-maigres.

Démonstration. Si $\Pi_{X}[A \cap(F \times G)]$ n'est pas co-maigre, soit $U$ un ouvert non vide tel que

$$
M:=\Pi_{X}[A \cap(F \times G)] \cap U
$$

soit maigre ; $A \cap(U \times Y)$ est $G_{\delta}$ 1.p.o. non vide de $U \times Y$, donc par le lemme 4.4 de [Le1] rencontre $(U \cap F \backslash M) \times G$ en un point $(x, y)$ qui vérifie $x \in M \backslash M$.

Théorème 1.13 Soient $X$ et $Y$ des espaces polonais parfaits de dimension $0, A$ un $G_{\delta}$ l.p.o. non vide de $X \times Y$. Alors il existe des ensembles presque-ouverts non vides $F$ et $G$, l'un contenu dans $X$ et l'autre dans $Y$, et une surjection continue ouverte de $F$ sur $G$ dont le graphe est contenu dans $A$ ou dans $\{(y, x) \in Y \times X /(x, y) \in A\}$ selon le cas.

\section{Démonstration.}

Premier cas. Dans tout rectangle ouvert non vide $U \times V$ tel que les projections de $A \cap(U \times V)$ soient denses dans $U$ et $V$, on trouve un sous-rectangle $U^{\prime} \times V^{\prime}$ de $U \times V$ ayant ces propriétés et tel que pour toute partie rare $R$ de $V^{\prime}, \Pi_{X}\left[A \cap\left(U^{\prime} \times R\right)\right]$ n'est pas dense dans $U^{\prime}$.

Soient $\left(O_{n}\right)$ une suite d'ouverts de $X \times Y$ telle que $A=\bigcap_{n \in \omega} O_{n}$, et $U_{\emptyset}$ et $V_{\emptyset}$ fournis par la propriété précédente appliquée aux projections de $A$. 
On construit alors des suites d'ouverts non vides $\left(U_{s}\right)_{s \in \omega<\omega}$ et $\left(V_{s}\right)_{s \in \omega<\omega}$ vérifiant

(i) $\bigcup_{n \in \omega} U_{s \frown n}$ est dense dans $U_{s}$

(ii) $U_{s} \times V_{s} \subseteq O_{|s|-1}$ si $s \neq \emptyset$

(iii) $\delta\left(U_{s}\right), \delta\left(V_{s}\right)<|s|^{-1}$ si $s \neq \emptyset$

(iv) $U_{s \frown n} \cap U_{s \frown m}=\emptyset$ si $n \neq m$

(v) $\overline{U_{s \frown n}} \subseteq U_{s}, \overline{V_{s \frown n}} \subseteq V_{s}$

(vi) Les projections de $A \cap\left(U_{s} \times V_{s}\right)$ sont denses dans $U_{s}$ et $V_{s}$

(vii) Si $R$ est rare dans $V_{s}, \Pi_{X}\left[A \cap\left(U_{s} \times R\right)\right]$ n'est pas dense dans $U_{s}$

Admettons la construction effectuée pour $|s| \leq p$. Partitionnons $\Pi_{X}\left[A \cap\left(U_{s} \times V_{s}\right)\right]$ (éventuellement privé d'un point) en une infinité d'ouverts-fermés non vides, disons $\left(Z_{n}\right)$, et soit

$$
T_{n}:=\Pi_{Y}\left[A \cap\left(Z_{n} \times V_{s}\right)\right]
$$

(c'est un ouvert non vide de $V_{s}$ ). Soit $H_{n}$ l'ensemble des parties de $\Sigma_{1}^{0}\left\lceil Z_{n} \backslash\{\emptyset\} \times \Sigma_{1}^{0}\left\lceil Z_{n} \backslash\{\emptyset\}\right.\right.$ telles que si $(U, V)$ et $\left(U^{\prime}, V^{\prime}\right)$ sont distincts dans $P, U$ et $U^{\prime}$ soient disjoints et $U \times V \subseteq O_{|s|}, \delta(U)$, $\delta(V)<(|s|+1)^{-1}, \bar{U} \subseteq Z_{n}, \bar{V} \subseteq T_{n}$, les projections de $A \cap(U \times V)$ soient denses dans $U$ et $V$, et pour toute partie rare $R$ de $V, \Pi_{X}[A \cap(U \times R)]$ ne soit pas dense dans $U$.

Alors $H_{n}$ n'est pas vide, puisqu'il contient le vide, et est ordonné de façon inductive par l'inclusion, donc par le lemme de Zorn a un élément maximal $P_{n}$. Alors si $P_{n}:=\left\{\left(U_{m}, V_{m}\right) / m \in I_{n}\right\}$, l'ouvert $u:=\bigcup_{m \in I_{n}} U_{m}$ est dense dans $Z_{n}$, sinon soit $U$ un ouvert non vide de $Z_{n}$ disjoint de $u$, et $(x, y)$ dans $A \cap\left(U \times T_{n}\right)$; on trouve des ouverts-fermés $U^{\prime \prime}$ et $V^{\prime \prime}$, de diamètre au plus $(|s|+1)^{-1}$, tels que $(x, y) \in U^{\prime \prime} \times V^{\prime \prime} \subseteq O_{|s|} \cap\left(U \times T_{n}\right)$. On applique alors la propriété du premier cas aux projections de $A \cap\left(U^{\prime \prime} \times V^{\prime \prime}\right)$ pour obtenir la contradiction cherchée avec la maximalité de $P$.

On obtient maintenant les $U_{s \frown n}$ en renumérotant la suite des ouverts $U$ pour lesquels on trouve $V$ et $n$ tels que $(U, V) \in P_{n}$, et $V_{s \frown n}$ est le $V$ correspondant. La construction est donc possible.

Soit $F^{\prime}:=\bigcap_{n \in \omega} \bigcup_{s \in \omega^{n}} U_{s}$; alors $F^{\prime}$ est $G_{\delta}$ dense de $U_{\emptyset}$, donc presque-ouvert. Si $x$ est dans $F^{\prime}$, on définit $f(x)$ de la manière habituelle, et la fonction $f$ est continue et uniformise partiellement $A$. De plus, si un ouvert non vide de $F^{\prime}$ avait une image maigre, il contiendrait un ouvert non vide d'image rare par le théorème de Baire et la continuité de $f$. Mais ceci est exclus à cause de la condition (vii), puisque $f\left[F^{\prime} \cap U_{s}\right] \subseteq V_{s}$.

Soit $\left(U_{n}\right)$ une base de la topologie de $F^{\prime},\left(V_{n}\right)$ une suite d'ouverts de $Y$, et $M_{n}$ une suite de $F_{\sigma}$ maigres de $Y$ tels que $f\left[F^{\prime} \cap U_{n}\right] \Delta V_{n} \subseteq M_{n}$. Posons $F=F^{\prime} \backslash\left(\bigcup_{n \in \omega} f^{-1}\left(M_{n}\right)\right) ; F$ est $G_{\delta}$ dense de $U_{\emptyset}$ comme $F^{\prime}$ par ce qui précède. Il est maintenant clair qu'on peut poser

$$
G:=\left(\bigcup_{n \in \omega} V_{n}\right) \backslash\left(\bigcup_{n \in \omega} M_{n}\right)
$$

Second cas. Il existe un rectangle ouvert non vide $U_{\emptyset} \times V_{\emptyset}$ tel que les projections de $A \cap\left(U_{\emptyset} \times V_{\emptyset}\right)$ soient denses dans $U_{\emptyset}$ et $V_{\emptyset}$, tel que pour tout sous-rectangle $U^{\prime} \times V^{\prime}$ de $U_{\emptyset} \times V_{\emptyset}$ ayant ces propriétés, on trouve une partie rare $R$ de $V^{\prime}$ telle que $\Pi_{X}\left[A \cap\left(U^{\prime} \times R\right)\right]$ soit dense dans $U^{\prime}$. 
Soient $n_{0} \in \omega, \varepsilon>0$, et $U^{\prime}$ et $V^{\prime}$ des ouverts ayant ces propriétés ; on montre qu'il existe des suites d'ouverts non vides $\left(U_{n}\right)$ et $\left(V_{n}\right)$ telles que

(i) $\bigcup_{n \in \omega} U_{n}$ est dense dans $U^{\prime}, \bigcup_{n \in \omega} V_{n}$ est dense dans $V^{\prime}$

(ii) $U_{n} \times V_{n} \subseteq O_{n_{0}}$

(iii) $\delta\left(U_{n}\right), \delta\left(V_{n}\right)<\varepsilon$

(iv) $V_{n} \cap V_{m}=\emptyset$ si $n \neq m$

(v) $\overline{U_{n}} \subseteq U^{\prime}, \overline{V_{n}} \subseteq V^{\prime}$

(vi) Les projections de $A \cap\left(U_{n} \times V_{n}\right)$ sont denses dans $U_{n}$ et $V_{n}$

Soit $\left(x_{n}\right)$ une suite dense de $\Pi_{X}\left[A \cap\left(U^{\prime} \times V^{\prime}\right)\right]$ (donc de U'). On va commencer par construire des suites d'ouverts-fermés non vides $\left(Z_{n}\right)$ et $\left(T_{n}\right)$ vérifiant $A \cap\left(Z_{n} \times T_{n}\right) \neq \emptyset$,

$$
Z_{n} \times T_{n} \subseteq O_{n_{0}} \cap\left[\left(U^{\prime} \cap \mathcal{B}\left(x_{n}, 2^{-n}\right)\right) \times V^{\prime} \backslash\left(\bigcup_{p<n} T_{p} \cup R\right)\right]
$$

de diamètre au plus $\varepsilon$.

Admettons avoir trouvé $\left(Z_{p}\right)_{p<n}$ et $\left(T_{p}\right)_{p<n}$ ayant ces propriétés. Comme $R$ est rare et non vide, $\bigcup_{p<n} T_{p} \subset_{\neq} V^{\prime} \backslash R$, et les projections de $A \cap\left[U^{\prime} \times\left(V^{\prime} \backslash\left(\bigcup_{p<n} T_{p}\right)\right)\right]$ sont co-maigres dans $U^{\prime}$ et $V^{\prime} \backslash\left(\bigcup_{p<n} T_{p}\right)$. Par le lemme précédent, celles de $A_{n}:=A \cap\left[U^{\prime} \times\left(V^{\prime} \backslash\left(\bigcup_{p<n} T_{p} \cup R\right)\right)\right]$ le sont également ; la projection sur $X$ rencontre donc $\mathcal{B}\left(x_{n}, 2^{-n}\right)$ en $z_{n}$; soit $y_{n}$ tel que $\left(z_{n}, y_{n}\right) \in A_{n}$, et $Z_{n}, T_{n}$ des ouverts-fermés de diamètre au plus $\varepsilon$ tels que l'on ait

$$
\left(z_{n}, y_{n}\right) \in Z_{n} \times T_{n} \subseteq O_{n_{0}} \cap\left[\left(U^{\prime} \cap \mathcal{B}\left(x_{n}, 2^{-n}\right)\right) \times V^{\prime} \backslash\left(\bigcup_{p<n} T_{p} \cup R\right)\right]
$$

La construction est donc possible.

Si $\bigcup_{n \in \omega} \Pi_{Y}\left[A \cap\left(Z_{n} \times T_{n}\right)\right]$ est dense dans $V^{\prime}$, on définit $V_{n}:=\Pi_{Y}\left[A \cap\left(Z_{n} \times T_{n}\right)\right]$ ainsi que

$$
U_{n}:=\Pi_{X}\left[A \cap\left(Z_{n} \times V_{n}\right)\right],
$$

et les conditions sont vérifiées, par densité de la suite $\left(z_{n}\right)$ dans $U^{\prime}$. Sinon, la construction précédente montre que les autres conditions sont réalisées. On pose

$$
Y^{\prime}:=\Pi_{Y}\left[A \cap\left(U^{\prime} \times V^{\prime}\right)\right] \backslash \overline{\bigcup_{n \in \omega} \Pi_{Y}\left[A \cap\left(Z_{n} \times T_{n}\right)\right]}
$$

Si $(x, y) \in A \cap\left(U^{\prime} \times Y^{\prime}\right)$, soit $Z_{x, y} \times T_{x, y}$ un rectangle ouvert de diamètre au plus $\varepsilon$ tel que l'on ait les inclusions $(x, y) \in Z_{x, y} \times T_{x, y} \subseteq \overline{Z_{x, y} \times T_{x, y}} \subseteq O_{n_{0}} \cap\left(U^{\prime} \times Y^{\prime}\right)$ et tel que les projections de $A \cap\left(Z_{x, y} \times T_{x, y}\right)$ soient $Z_{x, y}$ et $T_{x, y}$. On a $Y^{\prime}=\bigcup_{x, y} T_{x, y}=\bigcup_{n \in \omega} T_{x_{n}, y_{n}}$. Réduisons la suite $\left(T_{x_{n}, y_{n}}\right)$ en $\left(T_{n}^{\prime}\right)$, et posons $Z_{n}^{\prime}:=\Pi_{X}\left[A \cap\left(Z_{x_{n}, y_{n}} \times T_{n}^{\prime}\right)\right]$. Alors en ne gardant que les $T_{n}^{\prime}$ non vides, les $Z_{n}^{\prime}$ correspondants, et les $Z_{n}$ et $T_{n}$, on a ce qu'on veut. 
On recopie alors quasiment la construction du théorème 5.2 de [Le1] : soit $\phi_{0}$ de $\omega$ dans $\{\emptyset\}$ et, si $n>0, \phi_{n}$ une bijection de $\omega$ sur $\omega^{n}$. On construit une suite $\left(U_{s}\right)_{s \in \omega<\omega}$ d'ouverts non vides de $X$, et une suite $\left(V_{s}\right)_{s \in \omega<\omega}$ d'ouverts non vides de $Y$ vérifiant

(i) $\bigcup_{n \in \omega} \overline{U_{s \frown n}}$ est dense dans $U_{s}, \bigcup_{n \in \omega} \overline{V_{s \frown n}}$ est dense dans $V_{s}$

(ii) $U_{s} \times V_{s} \subseteq O_{|s|-1}$ si $s \neq \emptyset$

(iii) $\delta\left(U_{s}\right), \delta\left(V_{s}\right)<|s|^{-1}$ si $s \neq \emptyset$

(iv) $V_{s \frown n} \cap V_{s \frown m}=\emptyset$ si $n \neq m$

(v) $\left(\bigcup_{k \in \omega} U_{\phi_{n}(k)}\right) \cap \bigcup_{q+p<n}\left[U_{\phi_{q}(p)} \backslash\left(\bigcup_{l \in \omega} U_{\phi_{q}(p)-l}\right)\right]=\emptyset$

(vi) Les projections de $A \cap\left(U_{s} \times V_{s}\right)$ sont denses dans $U_{s}$ et $V_{s}$

Admettons avoir construit les suites $\left(U_{s}\right)_{|s| \leq n}$ et $\left(U_{s}\right)_{|s| \leq n},\left(U_{\phi_{n}(p)-k}\right)_{p<m, k \in \omega},\left(V_{\phi_{n}(p)-k}\right)_{p<m, k \in \omega}$ vérifiant (i)-(vi).

On construit, si ce n'est déjà fait, $\left(U_{\phi_{n}(m)-k}\right)_{k \in \omega}$ et $\left(V_{\phi_{n}(m)-k}\right)_{k \in \omega}$ en appliquant ce qui précède à $\varepsilon=(n+1)^{-1}, V^{\prime}:=V_{\phi_{n}(m)}, U^{\prime}:=U_{\phi_{n}(m)} \backslash \overline{\bigcup_{q+p<n}\left[U_{\phi_{q}(p)} \backslash\left(\bigcup_{l \in \omega} U_{\phi_{q}(p)-l}\right)\right]}$.

Les conditions demandées sont vérifiées, la densité des projections de $A \cap\left(U^{\prime} \times V^{\prime}\right)$ dans $U^{\prime}$ et $V^{\prime}$, donc dans $U_{\phi_{n}(m)}$ et $V_{\phi_{n}(m)}$, ne posant pas de problème à cause du lemme précédent. On définit alors $F$ et $G$ comme dans le théorème 5.2 de [Le1] ; ce sont des $G_{\delta}$ denses de $V_{\emptyset}$ et $U_{\emptyset}$, donc des presque-ouverts non vides. On conclut alors comme dans le théorème 5.2 de [Le1].

Sous les hypothèses du théorème 1.13 , il est faux en général que $A$ est uniformisable sur un $G_{\delta}$ dense de sa projection par une application continue ouverte sur son image (bien que $A$ soit uniformisable par une application continue). En effet, on prend l'exemple du lemme 1.3, ce qui fournit $A_{0} \subseteq N_{(0)} \times \omega^{\omega}$; si $A_{1}$ est le graphe d'un homéomorphisme de $\omega^{\omega} \backslash N_{(0)}$ sur $\omega^{\omega}, A_{0} \cup A_{1}$ est fermé 1.p.o. de $\omega^{\omega} \times \omega^{\omega}$, de projections $\omega^{\omega}$. Raisonnons par l'absurde : il existe un $G_{\delta}$ dense $G \operatorname{de} \omega^{\omega}$ et une application $f$ continue sur $G$ et ouverte sur $f[G]$, qui uniformise $A$ partiellement. Alors $f\left[G \cap N_{(0)}\right]$ est $G_{\delta}$ rare, par construction de $A_{0}$.

Mais comme $f\left[G \backslash N_{(0)}\right]$ est $G_{\delta}$ dense de $\omega^{\omega}, f[G]$ aussi, donc $f\left[G \cap N_{(0)}\right]$ est ouvert non vide et rare de $f[G]$, ce qui est absurde. Cependant, l'uniformisation a lieu si $A$ est le graphe d'une surjection continue ouverte de $Y$ dans $X$ :

Proposition 1.14 Soient $X$ et $Y$ des espaces métrisables séparables de dimension $0, Y$ étant complet, et $f: Y \rightarrow X$ une surjection continue ouverte ; alors il existe un homéomorphisme $g$ de $X$ sur son image tel que $f \circ g=I d_{X}$.

Démonstration. On construit des suites d'ouverts-fermés $\left(U_{s}\right)_{s \in \omega<\omega}$ et $\left(V_{s}\right)_{s \in \omega}<\omega$ vérifiant

(i) $\bigcup_{n \in \omega} U_{s \frown n}=U_{s}$

(ii) $U_{s}=f\left[V_{s}\right]$

(iii) $\delta\left(U_{s}\right), \delta\left(V_{s}\right)<|s|^{-1}$ si $s \neq \emptyset$

(iv) $U_{s \frown n} \cap U_{s \frown m}=V_{s \frown n} \cap V_{s \frown m}=\emptyset$ si $n \neq m$

(v) $V_{s} \neg n \subseteq V_{s}$ 
On pose $U_{\emptyset}:=X, V_{\emptyset}:=Y$; si on a $U_{s}$ et $V_{s}$, on partitionne $U_{s}$ en une suite $\left(U_{n}\right)$ d'ouvertsfermés de diamètre au plus $(|s|+1)^{-1}$; on partitionne ensuite l'ouvert-fermé $V_{s} \cap f^{-1}\left(U_{n}\right)$ en une suite $\left(V_{m}^{n}\right)_{m}$ d'ouverts-fermés de diamètre au plus $(|s|+1)^{-1}$. La suite double d'ouverts $\left(f\left[V_{m}^{n}\right]\right)$ est ensuite réduite en $\left(W_{m}^{n}\right)$, et on renumérote les $W_{m}^{n}$, l'ouvert-fermé correspondant dans $Y$ étant $V_{m}^{n} \cap f^{-1}\left(W_{m}^{n}\right)$.

Cette construction étant faite, elle définit de la manière habituelle une application continue

$$
g: X \rightarrow Y \text {. }
$$

De plus, si $\alpha$ est tel que $x \in \bigcap_{n \in \omega} U_{\alpha\lceil n}$, on a $f(g(x)) \in \bigcap_{n \in \omega} f\left[V_{\alpha\lceil n}\right]=\bigcap_{n \in \omega} U_{\alpha\lceil n}=\{x\}$, d'où le fait que $f \circ g=\operatorname{Id}_{X}$ et l'injectivité de $g$. Enfin, par la condition (ii), on a $g\left[U_{s}\right]=g[X] \cap V_{s}$, donc $g$ est ouverte sur son image.

Bien sûr, ce résultat est faux si on enlève la condition de dimension sur $X$.

Corollaire 1.15 Soient $X$ et $Y$ des espaces polonais parfaits de dimension 0, $A$ un $G_{\delta}$ l.p.o. non vide de $X \times Y$. Alors A est uniformisable sur un presque-ouvert non vide de $X$ par une application continue et ouverte sur son image.

Démonstration. On applique le théorème 1.13 : on a le résultat tout de suite ou alors on applique la proposition précédente.

A cause du lemme 1.3, l'image de l'application fournie par ce corollaire est en général rare.

\section{Applications aux classes de Wadge potentielles.}

On va maintenant appliquer les résultats d'uniformisation aux classes de Wadge potentielles : on obtient pour commencer des caractérisations des ensembles potentiellement différence d'ouverts parmi les ensembles potentiellement $\Sigma_{3}^{0}$ et $\Pi_{3}^{0}$ (donc par exemple parmi les boréliens à coupes dénombrables).

Lemme 2.1 Soit $X$ un espace polonais récursivement présenté. Alors il existe un $G_{\delta}$ dense $\Sigma_{1}^{1}$ de $X$ sur lequel la topologie de $X$ coïncide avec la topologie $\Delta$ engendrée par les $\Delta_{1}^{1}$.

Démonstration. Il est prouvé dans [Ke] que si $A$ est $\Delta_{1}^{1}$, il existe un $\Delta_{1}^{1}$ ouvert $U$ et un $\Delta_{1}^{1}$ à coupes fermées rares $F$ de $\omega \times X$ tels que $A \Delta U \subseteq \bigcup_{p \in \omega} F_{p}$. Cette propriété s'écrit de manière $\Pi_{1}^{1}$; on peut donc associer, à tout entier $n$ codant un $\Delta_{1}^{1}$, un entier $(f(n))_{0}$ codant un ouvert $\Delta_{1}^{1}$, et un entier $(f(n))_{1}$ codant un $\Delta_{1}^{1}$ à coupes fermées rares de $\omega \times X$, en gardant l'inclusion ci-dessus, et ce par une fonction $\Pi_{1}^{1}$-récursive partielle $f$. Désignons par $W^{X}$ un ensemble $\Pi_{1}^{1} \subseteq \omega$ de codes pour les $\Delta_{1}^{1}$ de $X$ et par $C^{X} \subseteq \omega \times X$ un ensemble $\Pi_{1}^{1}$ dont les sections aux points de $W^{X}$ décrivent les $\Delta_{1}^{1}$ de $X$ (cf [Lo2]). 
On pose $x \in G \Leftrightarrow \forall n \forall p n \notin W^{X}$ ou $\left((f(n))_{1}, p, x\right) \notin C^{\omega \times X}$. Alors $G$ répond au problème, car $\breve{G}$ est réunion dénombrable de fermés rares, et si $n$ code $A \in \Delta_{1}^{1}$, on a $A \cap G=C_{(f(n))_{0}}^{X} \cap G$, qui est ouvert dans $G$.

Dans la suite, si $f_{s}$ est une fonction partielle de $X$ dans $Y$ ou de $Y$ dans $X$, on notera $G\left(f_{s}\right)$ la partie de $X \times Y$ égale au graphe de $f_{s}$ si $f_{s}$ va de $X$ dans $Y$, et à $\operatorname{Gr}\left(f_{s}\right)^{*}:=\left\{(x, y) / x=f_{s}(y)\right\}$ sinon.

Lemme 2.2 Soient $X$ un espace polonais parfait récursivement présenté, $G$ un universel pour les $\Pi_{1}^{1}$ de $X, \varphi$ et $\psi$ des $\Pi_{1}^{1}$-opérateurs monotones sur $X$, et $\Phi^{\xi}$ les opérateurs sur $X$ définis par $\Phi^{0}(A)=A$, et $\Phi^{\xi}(A)=\varphi\left(\bigcup_{\eta<\xi} \Phi^{\eta}(A)\right)$ si $\xi$ est impair, $\Phi^{\xi}(A)=\psi\left(\bigcup_{\eta<\xi} \Phi^{\eta}(A)\right)$ si $\xi>0$ est pair. Alors si $R(\alpha, \beta, x) \Leftrightarrow \alpha \in W O$ et $x \in \Phi^{|\alpha|}\left(G_{\beta}\right)$, la relation $R$ est $\Pi_{1}^{1}$.

Démonstration. Ce lemme est démontré dans [Lo1] si $\varphi=\psi=\Phi$. La démonstration ici est analogue. Précisons-en les différences.

On sait qu'il existe une fonction $\Delta_{1}^{1}$-récursive $g: \omega^{\omega} \times \omega \rightarrow \omega^{\omega}$ telle que si $\alpha \in W O, g(\alpha, n)$ est dans $W O$ et code l'odre $\leq_{\alpha}$ restreint aux prédécesseurs de $n$ pour $\leq_{\alpha}$. On définit par récurrence une fonction $\Delta_{1}^{1}$-récursive $\varepsilon: \omega^{\omega} \rightarrow \omega$ telle que si $\alpha \in W O$, on ait l'égalité

$$
\varepsilon(\alpha)=\left\{\begin{array}{l}
0 \text { si }|\alpha| \text { est pair, } \\
1 \text { si }|\alpha| \text { est impair. }
\end{array}\right.
$$

On définit alors un $\Pi_{1}^{1}$-opérateur monotone $\Psi$ par

$$
(n, \alpha, \beta, x) \in \Psi(P) \Leftrightarrow\left\{\begin{array}{l}
(n=0 \text { et } \alpha \in W O \text { et } \forall p(0, g(\alpha, p), \beta, x) \in P) \text { ou } \\
\left(n=1 \text { et } \alpha \in W O \text { et } \exists q / q \leq_{\alpha} q \text { et } \forall p(0, g(\alpha, p), \beta, x) \in P\right. \text { et } \\
{[(\varepsilon(\alpha)=0 \text { et } x \in \psi(\{y \in X / \exists q P(1, g(\alpha, q), \beta, y)\})) \text { ou }} \\
(\varepsilon(\alpha)=1 \text { et } x \in \varphi(\{y \in X / \exists q P(1, g(\alpha, q), \beta, y)\}))]) \text { ou } \\
P(n, \alpha, \beta, x)
\end{array}\right.
$$

On montre alors comme dans [Lo1] que

$$
\begin{aligned}
& (0, \alpha, \beta, x) \in \Psi^{\xi}\left(P_{0}\right) \Leftrightarrow \alpha \in W O \text { et }|\alpha| \leq \xi \text {, et } \\
& (1, \alpha, \beta, x) \in \Psi^{\xi}\left(P_{0}\right) \Leftrightarrow \alpha \in W O \text { et }|\alpha| \leq \xi \text { et } x \in \Phi^{|\alpha|}\left(G_{\beta}\right) \text {, où }
\end{aligned}
$$

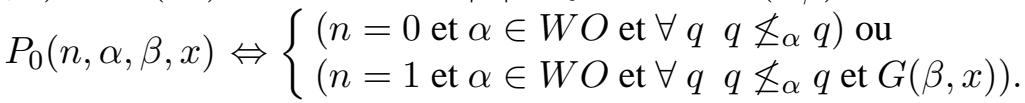

Comme l'opérateur $\Psi$ et $P_{0}$ sont $\Pi_{1}^{1}$, on a donc que $\Psi^{\infty}\left(P_{0}\right)$ est $\Pi_{1}^{1}$, et aussi que $(1, \alpha, \beta, x)$ est dans $\Psi^{\infty}\left(P_{0}\right)$ ssi $\left(\alpha \in W O\right.$ et $\left.x \in \Phi^{|\alpha|}\left(G_{\beta}\right)\right)$. On a donc le résultat, puique $R=\Psi^{\infty}\left(P_{0}\right)_{1}$.

Soit $\xi$ un ordinal dénombrable non nul. On définit $f: \omega^{<\omega} \rightarrow\{-1\} \cup(\xi+1)$, par récurrence sur $|s|$, comme suit : $f(\emptyset)=\xi$ et

$$
f(s \frown n)=\left\{\begin{array}{l}
\bullet-1 \text { si } f(s) \leq 0, \\
\bullet \theta \text { si } f(s)=\theta+1, \\
\bullet \text { un ordinal impair de } f(s) \text { tel que la suite }(f(s \frown n))_{n} \text { soit co-finale dans } \\
f(s) \text { et strictement croissante si } f(s) \text { est limite non nul. }
\end{array}\right.
$$

On définit alors des arbres : $T_{\xi}:=\left\{s \in \omega^{<\omega} / f(s) \neq-1\right\}$ et $T_{\xi}^{\prime}:=\left\{s \in T_{\xi} / f(s) \neq 0\right\}$. 
Théorème 2.3 Soient $X$ et $Y$ des espaces polonais, $A$ un borélien $\operatorname{pot}\left(\boldsymbol{\Sigma}_{3}^{0}\right) \cap \operatorname{pot}\left(\boldsymbol{\Pi}_{3}^{0}\right)$ de $X \times Y$, et $\xi$ un ordinal dénombrable.

(a) Si $\xi$ est pair non nul, A est non-pot $\left(D_{\xi}\left(\Sigma_{1}^{0}\right)\right)$ si et seulement s'il existe des espaces polonais parfaits $Z$ et $T$ de dimension 0 , des ouverts-fermés non vides $A_{s}$ et $B_{s}$ (l'un dans $Z$ et l'autre dans $T$, pour $s$ dans $T_{\xi}$ ), des surjections continues ouvertes $f_{s}$ de $A_{s}$ sur $B_{s}$, et des injections continues u et $v$ tels que si $B_{p}:=\bigcup_{s \in T_{\xi} /|s| \text { paire }} G\left(f_{s}\right)$ et $B_{i}:=\bigcup_{s \in T_{\xi} /|s| \text { impaire }} G\left(f_{s}\right)$, on ait $\overline{B_{p}}=B_{p} \cup B_{i}$, $B_{p} \subseteq(u \times v)^{-1}(A), B_{i} \subseteq(u \times v)^{-1}(\check{A})$, et $G\left(f_{s}\right)=\overline{\bigcup_{n \in \omega} G\left(f_{s \frown n}\right)} \backslash\left(\bigcup_{n \in \omega} G\left(f_{s \frown n}\right)\right)$ si $s \in T_{\xi}^{\prime}$.

(b) Si $\xi$ est impair, A est non-pot $\left(\check{D}_{\xi}\left(\boldsymbol{\Sigma}_{1}^{0}\right)\right)$ si et seulement s'il existe des espaces polonais $Z$ et $T$ parfaits de dimension 0 , des ouverts-fermés non vides $A_{s}$ et $B_{s}$ (l'un dans $Z$ et l'autre dans $T$, pour $s$ dans $T_{\xi}$ ), des surjections continues ouvertes $f_{s}$ de $A_{s}$ sur $B_{s}$, et des injections continues $u$ et $v$ tels que si $B_{p}:=\bigcup_{s \in T_{\xi} /|s| \text { paire }} G\left(f_{s}\right)$ et $B_{i}:=\bigcup_{s \in T_{\xi} /|s| \text { impaire }} G\left(f_{s}\right)$, on ait $\overline{B_{i}}=B_{p} \cup B_{i}$, $B_{i} \subseteq(u \times v)^{-1}(A), B_{p} \subseteq(u \times v)^{-1}(\check{A})$, et $G\left(f_{s}\right)=\overline{\bigcup_{n \in \omega} G\left(f_{s \frown n}\right)} \backslash\left(\bigcup_{n \in \omega} G\left(f_{s \frown n}\right)\right)$ si $s \in T_{\xi}^{\prime}$.

Démonstration. Montrons (a), la preuve de (b) étant analogue. Supposons que $A$ est $\operatorname{pot}\left(D_{\xi}\left(\boldsymbol{\Sigma}_{1}^{0}\right)\right)$, en raisonnant par l'absurde ; alors $B_{p}=\overline{B_{p}} \cap(u \times v)^{-1}(A)$, l'est aussi, cette classe étant stable par intersection avec les fermés, et on trouve un $G_{\delta}$ dense $F$ (resp. $G$ ) de $Z$ (resp. $T$ ) tels que $B_{p} \cap(F \times G)$ soit $D_{\xi}\left(\Sigma_{1}^{0}\right)$ dans $F \times G$. On trouve donc une suite croissante d'ouverts de $F \times G$, disons $\left(U_{\eta}\right)_{\eta<\xi}$, telle que $B_{p} \cap(F \times G)=\bigcup_{\eta<\xi, \eta}$ impair $U_{\eta} \backslash\left(\bigcup_{\theta<\eta} U_{\theta}\right)$.

Montrons que si $\eta \leq \xi, s \in T_{\xi}$ et $f(s)=\eta$, alors $G\left(f_{s}\right) \cap(F \times G) \subseteq \check{U}_{\eta}$ si $\eta<\xi$, et $G\left(f_{s}\right) \cap(F \times G) \subseteq{ }^{c}\left(\bigcup_{\theta<\eta} U_{\theta}\right)$ si $\eta=\xi$. On aura la contradiction cherchée avec $s=\emptyset$ et $\eta=\xi$.

On procède par récurrence sur $\eta$. Remarquons que si $s$ est dans $T_{\xi},|s|$ est paire si et seulement si $f(s)$ est pair. Si $\eta=0,|s|$ est paire, donc $G\left(f_{s}\right) \cap(F \times G) \subseteq B_{p} \cap(F \times G) \subseteq \check{U}_{0}$.

Admettons le résultat pour $\theta<\eta$. Si $\eta$ est le successeur de $\theta$, par hypothèse de récurrence on a $G\left(f_{s \frown m}\right) \cap(F \times G) \subseteq \check{U}_{\theta}$ pour tout $m$; d'où, si on pose $C_{s}:=\bigcup_{n \in \omega} G\left(f_{s \frown n}\right)$,

$$
C_{s} \cap(F \times G) \subseteq \check{U}_{\theta}
$$

et ${\overline{C_{s} \cap(F \times G)}}^{F \times G} \subseteq \check{U}_{\theta}$. Mais comme dans la preuve du lemme 3.5 de [Le1], on a

$$
{\overline{C_{s} \cap(F \times G)}}^{F \times G}=\overline{C_{s}} \cap(F \times G),
$$

d'où l'inclusion cherchée si $\eta=\xi$.

Si $\eta<\xi$ et $|s|$ est paire, $f(s)$ est pair et $\theta$ est impair. On a les inclusions successives

$$
G\left(f_{s}\right) \cap(F \times G) \subseteq B_{p} \cap(F \times G) \subseteq \bigcup_{\varepsilon<\xi, \varepsilon \text { impair }} U_{\varepsilon} \backslash\left(\bigcup_{\gamma<\varepsilon} U_{\gamma}\right) \subseteq \check{U}_{\theta+1} .
$$

Si $|s|$ est impaire, $f(s)$ est impair et $\theta$ est pair. Mais si $s \in T_{\xi}^{\prime}$ est de longueur impaire, on a

$$
G\left(f_{s}\right) \cap(F \times G) \subseteq(F \times G) \backslash B_{p}=(F \times G) \backslash\left(\bigcup_{\varepsilon<\xi} U_{\varepsilon}\right) \cup \bigcup_{\varepsilon<\xi, \varepsilon \text { pair }} U_{\varepsilon} \backslash\left(\bigcup_{\gamma<\varepsilon} U_{\gamma}\right),
$$

ceci parce que $G\left(f_{s}\right) \cap B_{p}=\emptyset$. On a donc le résultat en appliquant l'hypothèse de récurrence. 
Si $\eta$ est un ordinal limite, $(f(s \frown n))_{n}$ est co-finale dans $f(s)$, donc par hypothèse de récurrence, on a $G\left(f_{s \frown n}\right) \cap(F \times G) \subseteq \check{U}_{f(s \frown n)}$. Si $\theta_{0}<f(s)$, on trouve $n\left(\theta_{0}\right)$ tel que $f(s \frown n)>\theta_{0}$ si $n\left(\theta_{0}\right) \leq n$. Donc $G\left(f_{s \frown n}\right) \cap(F \times G) \subseteq \check{U}_{\theta_{0}}$ dès que $n\left(\theta_{0}\right) \leq n$. Or

$G\left(f_{s}\right) \cap(F \times G) \subseteq(F \times G) \cap \overline{C_{s}} \backslash C_{s}={\overline{(F \times G) \cap C_{s}}}^{F \times G} \backslash C_{s} \subseteq{\overline{\bigcup_{n\left(\theta_{0}\right) \leq n}(F \times G) \cap G\left(f_{s} \frown n\right)}}^{F \times G} \subseteq \check{U}_{\theta_{0}}$.

Donc $G\left(f_{s}\right) \cap(F \times G) \subseteq{ }^{c}\left(\bigcup_{\theta<\eta} U_{\theta}\right)$. Si $\eta<\xi$, comme $|s|$ est paire, on a l'inclusion

$$
G\left(f_{s}\right) \cap(F \times G) \subseteq \bigcup_{\varepsilon<\xi, \varepsilon \text { impair }} U_{\varepsilon} \backslash\left(\bigcup_{\gamma<\varepsilon} U_{\gamma}\right),
$$

$\operatorname{donc} G\left(f_{s}\right) \cap(F \times G) \subseteq \check{U}_{\eta}$.

Inversement, soit $A$ dans $\operatorname{pot}\left(\boldsymbol{\Sigma}_{3}^{0}\right) \cap \operatorname{pot}\left(\boldsymbol{\Pi}_{3}^{0}\right) \backslash \operatorname{pot}\left(D_{\xi}\left(\boldsymbol{\Sigma}_{1}^{0}\right)\right)$ dans $X \times Y$, avec $X$ et $Y$ polonais. Nécessairement $X$ et $Y$ sont non dénombrables, donc boréliennement isomorphes à $\omega^{\omega}$, disons par $\varphi$ et $\psi$. Remarquons qu'on peut supposer $X$ et $Y$ parfaits. Soit alors $\beta$ tel que $\xi<\omega_{1}^{\beta}$, tel que $X$ et $Y$ soient récursivement en $\beta$-présentés, tel que $\varphi$ et $\psi$ soient $\Delta_{1}^{1}(\beta)$, et tel que $A$ et $\check{A}$ soient réunion d'une $\Delta_{1}^{1}(\beta)$-suite de $G_{\delta}$ pour le produit $\Delta_{X}^{\beta} \times \Delta_{Y}^{\beta}$ (où $\Delta_{X}^{\beta}$ est la topologie engendrée par les $\Delta_{1}^{1}(\beta)$ de $X)$. Posons $\Omega_{X}^{\beta}:=\left\{x \in X / \omega_{1}^{(\varphi(x), \beta)} \leq \omega_{1}^{\beta}\right\}, D_{X}^{\beta}:=\left\{x \in X / x \notin \Delta_{1}^{1}(\beta)\right\}, Z_{0}:=\Omega_{X}^{\beta} \cap D_{X}^{\beta}$, $T_{0}:=\Omega_{Y}^{\beta} \cap D_{Y}^{\beta}$.

Ces espaces $Z_{0}$ et $T_{0}$, si on les munit des restrictions des topologies de Gandy-Harrington $\Sigma_{X}^{\beta}$ (resp. $\Sigma_{X}^{\beta}$ ), sont polonais parfaits de dimension 0 . En effet, $Z_{0}$ et $T_{0}$ sont $\Sigma_{1}^{1}(\beta)$ comme intersection de deux $\Sigma_{1}^{1}(\beta)$ (cf [Mo]). Ceci prouve qu'ils n'ont pas de point isolé. De plus, si $E$ est $\Sigma_{1}^{1}(\beta)$ contenu dans $\Omega_{X}^{\beta}, E$ est ouvert-fermé dans $\Omega_{X}^{\beta}$ pour la restriction de $\Sigma_{X}^{\beta}$ : on a, si $f$ est $\Delta_{1}^{1}(\beta)$ telle que $x \notin E \Leftrightarrow f(x) \in W O$,

$$
x \in \Omega_{X}^{\beta} \backslash E \Leftrightarrow \exists \xi<\omega_{1}^{\beta}(f(x) \in W O \text { et }|f(x)| \leq \xi) \text { et } x \in \Omega_{X}^{\beta} .
$$

On en déduit que $\Omega_{X}^{\beta}$ est à base dénombrable d'ouverts-fermés, donc métrisable séparable ; comme il est ouvert d'un espace fortement $\alpha$-favorable, il est lui-même fortement $\alpha$-favorable, donc polonais (cf [Lo1] pour plus de détails).

On définit $\Omega_{X \times Y}^{\beta}:=\left\{(x, y) \in X \times Y / \omega_{1}^{(\varphi(x), \psi(y), \beta)} \leq \omega_{1}^{\beta}\right\}$; alors on sait que $\Omega_{X \times Y}^{\beta}$ rencontre tout ensemble $\Sigma_{1}^{1}(\beta)$ non vide de $X \times Y$ (cf [Lo1]).

On définit ensuite par récurrence

$$
F_{\eta}:=\left\{\begin{array}{l}
\overline{A \cap \bigcap_{\theta<\eta} F_{\theta}} \text { si } \eta \text { est pair, } \\
\overline{A \cap \bigcap_{\theta<\eta} F_{\theta}} \text { si } \eta \text { est impair, }
\end{array}\right.
$$

l'adhérence étant prise au sens de la topologie $\Delta_{X}^{\beta} \times \Delta_{Y}^{\beta}$. 
Montrons que les $F_{\eta}$ sont $\Sigma_{1}^{1}(\beta)$ pour $\eta \leq \xi$. On définit des $\Pi_{1}^{1}(\beta)$-opérateurs monotones sur $X \times Y$ par les formules : $\varphi(P)=P \cup \operatorname{Int}(P \cup A)$ et $\psi(P)=P \cup \operatorname{Int}(P \cup \check{A})$, où l'intérieur est pris au sens de la topologie $\Delta_{X}^{\beta} \times \Delta_{Y}^{\beta}$. Il est manifeste que, avec les notations du lemme 2.2 , on a $\check{F}_{\eta}=\Phi^{\eta}(\check{\bar{A}})$ si $\eta$ est dénombrable, par récurrence transfinie. Il suffit alors d'appliquer ce lemme 2.2.

Montrons que si $C$ et $D$ sont co-dénombrables, alors $F_{\xi} \cap(C \times D) \neq \emptyset$. Pour ce faire, posons $A^{\prime}:=A \cap(C \times D)$. Par la remarque 2.1 de [Le1], $A^{\prime}$ est non-pot $\left(D_{\xi}\left(\Sigma_{1}^{0}\right)\right)$. Soit $\gamma$ tel que $\beta, C$ et $D$ soient $\Delta_{1}^{1}(\gamma)$, et posons

$$
F_{\eta}^{\gamma}:=\left\{\begin{array}{l}
\overline{A^{\prime} \cap \bigcap_{\theta<\eta} F_{\theta}^{\gamma}} \text { si } \eta \text { est pair, } \\
\overline{A \cap \cap \bigcap_{\theta<\eta} F_{\theta}^{\gamma}} \text { si } \eta \text { est impair, }
\end{array}\right.
$$

l'adhérence étant prise au sens de la topologie $\Delta_{X}^{\gamma} \times \Delta_{Y}^{\gamma}$. Alors il est clair que si $\eta \leq \xi$,

$$
F_{\eta}^{\gamma} \subseteq F_{\eta} \cap(C \times D)
$$

ce par récurrence transfinie. Il suffit donc de voir que $F_{\xi}^{\gamma}$ est non vide. En raisonnant par l'absurde, on va montrer que si $U_{\eta}=\check{F}_{\eta}^{\gamma}$, alors $A^{\prime}=\bigcup_{\eta<\xi, \eta}$ impair $U_{\eta} \backslash\left(\bigcup_{\theta<\eta} U_{\theta}\right)$. Si $\eta$ est impair et successeur de $\theta_{0}, U_{\eta} \backslash\left(\bigcup_{\theta<\eta} U_{\theta}\right)=F_{\theta_{0}}^{\gamma} \backslash F_{\eta}^{\gamma}=F_{\theta_{0}}^{\gamma} \backslash \overline{\check{A} \cap F_{\theta_{0}}^{\gamma}} \subseteq A^{\prime}$. Si maintenant $x$ est dans $A^{\prime}, x$ est dans $U_{\xi}$ donc il existe un plus petit $\eta \leq \xi$ tel que $x$ soit dans $U_{\eta}$. Si $\eta$ est pair et successeur de $\theta_{0}, x$ est dans $U_{\eta} \backslash\left(\bigcup_{\theta<\eta} U_{\theta}\right)=F_{\theta_{0}}^{\gamma} \backslash F_{\eta}^{\gamma}=F_{\theta_{0}}^{\gamma} \backslash \overline{A^{\prime} \cap F_{\theta_{0}}^{\gamma}} \subseteq \check{A}^{\prime}$. Si $\eta$ est limite, $x$ est dans $\overline{A^{\prime} \cap \bigcap_{\theta<\eta} F_{\theta}^{\gamma}} \backslash\left(\bigcup_{\theta<\eta} U_{\theta}\right)=\overline{A^{\prime} \cap \bigcap_{\theta<\eta} F_{\theta}^{\gamma}} \cap\left(\bigcap_{\theta<\eta} F_{\theta}^{\gamma}\right) \subseteq \check{A}^{\prime}$. D'où le résultat.

On en déduit que $F_{\xi} \cap\left(D_{X}^{\beta} \times D_{Y}^{\beta}\right)$ est un $\Sigma_{1}^{1}(\beta)$ non vide, donc qu'il rencontre l'ensemble $\Omega_{X \times Y}^{\beta} \subseteq \Omega_{X}^{\beta} \times \Omega_{Y}^{\beta}$, et que $F_{\xi} \cap\left(Z_{0} \times T_{0}\right)$ est non vide. On remarque alors que dans la définition des $F_{\eta}$, pour $\eta \leq \xi$, on peut tout aussi bien prendre l'adhérence au sens de $\Sigma_{X}^{\beta} \times \Sigma_{Y}^{\beta}$ car les ensembles sous l'adhérence sont $\Sigma_{1}^{1}(\beta)$. On a donc que $F_{\xi} \cap\left(Z_{0} \times T_{0}\right)=\frac{\left.\overline{A \cap}_{0} \times T_{0}\right) \cap \bigcap_{\eta<\xi} F_{\eta}}{Z_{0} \times T_{0}}$. L'ensemble $A \cap\left(Z_{0} \times T_{0}\right) \cap \bigcap_{\eta<\xi} F_{\eta}$ est donc réunion de $G_{\delta}$ l.p.o. de $Z_{0} \times T_{0}$, et le théorème 1.13 peut s'appliquer à l'un de ces $G_{\delta}$ qui est non vide ; ce qui fournit des presque-ouverts non vides $a_{\emptyset}$ et $b_{\emptyset}$, ainsi que $g_{\emptyset}: a_{\emptyset} \rightarrow b_{\emptyset}$ surjective continue ouverte.

On construit alors, pour $s$ dans $T_{\xi}$, des suites $\left(a_{s}\right)$ et $\left(b_{s}\right)$ de presque-ouverts (l'un dans $Z_{0}$, l'autre dans $\left.T_{0}\right)$, une suite $\left(g_{s}\right)$ de surjections continues ouvertes de $a_{s}$ sur $b_{s}$, des suites denses $\left(x_{n}^{s}\right)_{n}$ de $a_{s}$ vérifiant :

(i) $G\left(g_{s}\right) \subseteq\left\{\begin{array}{l}\left(Z_{0} \times T_{0}\right) \cap \bigcap_{n \in \omega} F_{f(s \neg n)} \cap A \text { si }|s| \text { est paire }\left(\left(Z_{0} \times T_{0}\right) \cap A \text { si } f(s)=0\right), \\ \left(Z_{0} \times T_{0}\right) \cap \bigcap_{n \in \omega} F_{f(s \neg n)} \cap \check{A} \text { si }|s| \text { est impaire, }\end{array}\right.$

(ii) $G\left(g_{s \frown n}\right)\left(\right.$ ou $\left.G\left(g_{s \frown n}\right)^{*}\right) \subseteq \mathcal{B}\left[\left(x_{n}^{s}, g_{s}\left(x_{n}^{s}\right)\right), 2^{-\Sigma_{i \leq|s|}\left(s^{\frown}{ }^{\prime}(i)+1\right)}\right]$ si $s \in T_{\xi}^{\prime}$.

Admettons avoir construit $g_{s}$ pour $|s| \leq p$, et soient $s$ de longueur $p$, et $\left(x_{n}^{s}\right)_{n}$ une suite dense de $a_{s}$. Alors si $p$ est pair et $n$ entier, $G\left(g_{s}\right) \subseteq F_{f(s \frown n)} \cap\left(Z_{0} \times T_{0}\right)$, qui est égal à

$$
\left(Z_{0} \times T_{0}\right) \cap \overline{\bigcap_{m \in \omega} F_{f(s \frown n \frown m) \backslash A} .}
$$


D'où $G\left(g_{s}\right) \subseteq \overline{\left(Z_{0} \times T_{0}\right) \cap \bigcap_{m \in \omega} F_{f(s \frown n \frown m)} \backslash A}$. On peut alors appliquer le théorème 1.13 à l'un des ensembles $G_{\delta}$ et $\Sigma_{1}^{1}(\beta)$ non vides dont l'intersection

$$
\bigcap_{m \in \omega} F_{f(s \frown n \frown m)} \backslash A \cap \mathcal{B}\left[\left(x_{n}^{s}, g_{s}\left(x_{n}^{s}\right)\right), 2^{-\Sigma_{i \leq|s|}\left(s^{\frown} n(i)+1\right)}\right]
$$

(ou alors l'intersection $\left.\bigcap_{m \in \omega} F_{f(s \frown n \frown m)} \backslash A \cap \mathcal{B}\left[\left(g_{s}\left(x_{n}^{s}\right), x_{n}^{s}\right), 2^{-\Sigma_{i \leq|s|}\left(s^{\frown} n(i)+1\right)}\right]\right)$ est la réunion, dans le produit $Z_{0} \times T_{0}$, ce qui fournit le graphe recherché. De même si $p$ est impair.

On choisit alors $\alpha$ tel que $Z_{0}$ et $T_{0}$ soient récursivement en $\alpha$-présentés, et tel que pour tout $s \in T_{\xi}$ et pour tout $p \in \omega, \bigcup_{n \in \omega} G\left(g_{s} \frown n\right), G\left(g_{s}\right)$ et $\bigcup_{s \in \omega \leq p} G\left(g_{s}\right)$ soient $\Delta_{1}^{1}(\alpha)$. On prend des notations analogues aux précédentes. Soit $M_{0}$ (resp. $N_{0}$ ) un $G_{\delta}$ dense $\Sigma_{1}^{1}(\alpha)$ de $Z_{0}$ (resp. $T_{0}$ ), fourni par le lemme 2.1, sur lequel la topologie de $Z_{0}$ (resp. $T_{0}$ ) coïncide avec la topologie $\Delta_{Z_{0}}^{\alpha}$ (resp. $\Delta_{T_{0}}^{\alpha}$ ). On définit maintenant les objets recherchés :

$$
\begin{gathered}
Z:=M_{0} \cap \Omega_{Z_{0}}^{\alpha} \cap D_{Z_{0}}^{\alpha}, T:=N_{0} \cap \Omega_{T_{0}}^{\alpha} \cap D_{T_{0}}^{\alpha}, \\
G\left(f_{s}\right):=\left\{\begin{array}{l}
G\left(g_{s}\right) \cap(Z \times T) \text { si } f(s)=0, \\
G\left(g_{s}\right) \cap \overline{C_{s}} Z \times T \text { si } s \in T_{\xi}^{\prime} .
\end{array}\right.
\end{gathered}
$$

$A_{s}$ et $B_{s}$ sont les projections de $G\left(f_{s}\right)$, et $u$ (resp. $v$ ) est l'application identique de $Z$ dans $X$ (resp. $T$ dans $Y)$. Vérifions que ces objets conviennent. Montrons que $G\left(f_{s}\right)=G\left(g_{s}\right) \cap(Z \times T)$ si $s \in T_{\xi}$.

La relation est vraie par définition si $f(s)=0$. Admettons-la pour $f(s)<\eta \leq \xi$; soit $s \in T_{\xi}$ tel que $f(s)=\eta>0$. On a bien sûr que $G\left(f_{s}\right) \subseteq G\left(g_{s}\right) \cap(Z \times T)$. Mais on a

$$
G\left(f_{s}\right)=G\left(g_{s}\right) \cap{\overline{\bigcup_{n \in \omega} G\left(f_{s \frown n}\right)}}^{Z \times T}=G\left(g_{s}\right) \cap{\overline{\bigcup_{n \in \omega} G\left(g_{s}{ }_{n}\right) \cap(Z \times T)}}^{Z \times T},
$$

par hypothèse de récurrence. Montrons donc que $G\left(g_{s}\right) \cap(Z \times T) \subseteq{\overline{\bigcup_{n \in \omega} G\left(g_{s} \frown n\right) \cap(Z \times T)}}^{Z \times T}$. On a $G\left(g_{s}\right) \subseteq{\overline{\bigcup_{n \in \omega} G\left(g_{s \frown n}\right)}}^{Z_{0} \times T_{0}}$ car si $O$ est ouvert-fermé et contient $\left(x, g_{s}(x)\right)$, on trouve une suite strictement croissante $\left(n_{q}\right)_{q}$ telle que $\left(x_{n_{q}}^{s}\right)_{q}$ converge vers $x$. Par continuité de $g_{s}$, la suite image converge vers $g_{s}(x)$, et on trouve $q$ tel que $\mathcal{B}\left[\left(x_{n_{q}}^{s}, g_{s}\left(x_{n_{q}}^{s}\right)\right), 2^{-\Sigma_{i \leq|s|}\left(s^{\frown} n_{q}(i)+1\right)}\right] \subseteq O$, et $G\left(g_{s \frown n_{q}}\right) \subseteq O$ (ou $\left.G\left(g_{s \frown n_{q}}\right) \subseteq O^{*}\right)$. On a que $\Omega_{Z_{0}}^{\alpha}$ est co-maigre dans $Z_{0}$. En effet, $Z_{0}$ est polonais parfait de dimension 0 et non vide, donc on peut choisir l'isomorphisme avec $\omega^{\omega}$ de façon à préserver les ensembles co-maigres ; il suffit alors de consulter [Ke], où il est démontré que $\Omega_{\omega^{\omega}}^{\alpha}$ est co-maigre dans $\omega^{\omega}$. Donc $Z$ (resp. $T$ ) est co-maigre dans $Z_{0}$ (resp. $T_{0}$ ) et

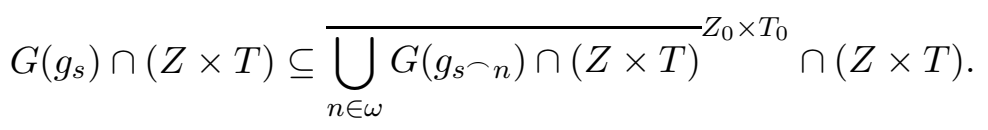

Or ce dernier ensemble est ${\overline{\bigcup_{n \in \omega} G\left(g_{s \frown n}\right) \cap(Z \times T)}}^{Z \times T}$, car sur $Z$ (resp. T), les topologies initiales et $\Delta_{Z_{0}}^{\alpha}$ (resp. $\Delta_{T_{0}}^{\alpha}$ ) coïncident, et car on a un $\Sigma_{1}^{1}(\alpha)$ sous l'adhérence, donc les adhérences pour $\Delta_{Z_{0}}^{\alpha} \times \Delta_{T_{0}}^{\alpha}$ et $\Sigma_{Z_{0}}^{\alpha} \times \Sigma_{T_{0}}^{\alpha}$ sont les mêmes. 
Comme $\Omega_{Z_{0}}^{\alpha} \cap D_{Z_{0}}^{\alpha}$, muni de la restriction de la topologie de Gandy-Harrington, est polonais parfait de dimension $0, Z$ l'est aussi puisqu'il en est un ouvert. De même pour $T$. Les seules choses non évidentes à vérifier sont que $G\left(f_{s}\right)$ n'est pas vide, l'inclusion $\overline{C_{s}} \backslash C_{s} \subseteq G\left(f_{s}\right)$, et aussi que $\overline{B_{p}}=B_{p} \cup B_{i}$.

L'ensemble $G\left(f_{s}\right)$ est non vide puisqu'il est égal à $G\left(g_{s}\right) \cap(Z \times T)$ et que $Z$ (resp. $T$ ) est comaigre dans $Z_{0}$ (resp. $T_{0}$ ).

Soit $(x, y)$ dans ${\overline{C_{s}}}^{Z \times T} \backslash C_{s},\left(\left(x_{n}, y_{n}\right)\right)$ dans $C_{s}$ convergeant vers $(x, y)$, et $p_{n}$ tel que $\left(x_{n}, y_{n}\right)$ soit dans $G\left(f_{s \frown p_{n}}\right)$. Comme $G\left(f_{s \frown p_{n}}\right)$ est fermé dans $Z \times T$, on peut supposer la suite $\left(p_{n}\right)$ strictement croissante. La distance de $\left(x_{n}, y_{n}\right)$ à $G\left(g_{s}\right)$, dans $Z_{0} \times T_{0}$, est au plus $2^{-\Sigma_{i \leq|s|}\left(s^{\frown} p_{n}(i)+1\right)}$, et comme la convergence a lieu aussi dans $Z_{0} \times T_{0}$, on a $(x, y) \in \overline{G\left(g_{s}\right)} Z_{0} \times T_{0}$.

Mais on a l'égalité $\overline{G\left(g_{s}\right)} Z_{0} \times T_{0} \cap(Z \times T)={\overline{G\left(g_{s}\right) \cap(Z \times T)}}^{Z_{0} \times T_{0}} \cap(Z \times T)$ car $Z$ et $T$ sont co-maigres, d'où $(x, y) \in{\overline{G\left(f_{s}\right)}}^{Z \times T}$, comme précédemment. Comme le graphe de $f_{s}$ est fermé, on a bien $(x, y) \in G\left(f_{s}\right)$.

Montrons par récurrence sur $p$ que $G\left(f_{\emptyset}\right) \cup \bigcup_{|s|<p, s \in T_{\xi}^{\prime}} C_{s}$ est fermé. C'est clair pour $p=0$. Admettons donc que c'est vrai pour $p$. On a les égalités

$$
G\left(f_{\emptyset}\right) \cup \bigcup_{|s|<p+1} C_{s}=G\left(f_{\emptyset}\right) \cup \bigcup_{|s|<p} C_{s} \cup \bigcup_{s \in \omega^{p}} C_{s}=G\left(f_{\emptyset}\right) \cup \bigcup_{|s|<p} C_{s} \cup \bigcup_{s \in \omega^{p+1}} G\left(f_{s}\right) .
$$

Soit $\left(\left(x_{m}, y_{m}\right)\right) \subseteq G\left(f_{\emptyset}\right) \cup \bigcup_{|s|<p+1} C_{s}$ convergeant vers $(x, y)$. Alors par hypothèse de récurrence, on peut supposer que pour chaque $m$ il existe $\left(s_{m}, n_{m}\right)$ tel que $\left(x_{m}, y_{m}\right)$ soit dans $G\left(f_{s_{\widehat{m}} n_{m}}\right)$. Les ensembles $G\left(f_{s \frown n}\right)$ étant fermés, on peut supposer que la suite $\left(\Sigma_{i \leq p}\left(s_{m} n_{m}(i)+1\right)\right)_{m}$ tend vers l'infini. La distance de $\left(x_{m}, y_{m}\right)$ à $G\left(g_{s_{m}}\right)$, dans $Z_{0} \times T_{0}$, est au plus $2^{-\Sigma_{i \leq p}\left(s_{\bar{m}} n_{m}(i)+1\right)}$, donc comme la convergence a lieu aussi dans $Z_{0} \times T_{0}$, on a

$$
(x, y) \in \overline{G\left(g_{\emptyset}\right) \cup \bigcup_{(s, n) \in \omega^{<p} \times \omega} G\left(g_{s \frown n}\right)} Z^{Z_{0} \times T_{0}} \cap(Z \times T) .
$$

Mais en raisonnant comme précédemment, on voit que

$$
(x, y) \in{\overline{G\left(f_{\emptyset}\right) \cup \bigcup_{s \in \omega<p} C_{s}}}^{Z \times T}=G\left(f_{\emptyset}\right) \cup \bigcup_{s \in \omega<p} C_{s},
$$

par hypothèse de récurrence. Donc $(x, y) \in G\left(f_{\emptyset}\right) \cup \bigcup_{s \in \omega<p+1} C_{s}$, qui est donc fermé.

On a que $G\left(f_{\emptyset}\right) \cup \bigcup_{s \in T_{\xi}^{\prime}} C_{s}$ est fermé. En effet, si $\left(\left(z_{m}, t_{m}\right)\right) \subseteq G\left(f_{\emptyset}\right) \cup \bigcup_{s \in T_{\xi}^{\prime}} C_{s}$ converge vers $(z, t)$, par ce qui précède on peut supposer que pour chaque $m$ il existe $s_{m}^{\prime}$ tel que $\left(z_{m}, t_{m}\right)$ soit dans $G\left(f_{s_{m}^{\prime}}\right)$, et que la suite $\left(\left|s_{m}^{\prime}\right|\right)_{m}$ tend vers l'infini. Alors on trouve $p$ tel que l'ensemble des $s_{m}^{\prime}(p)$ soit infini. 
En effet, si tel n'est pas le cas, $\left\{\begin{array}{ll}s \in T_{\xi} / \exists m & \left.s \prec s_{m}^{\prime}\right\}\end{array}\right.$ est un sous-arbre infini de $T_{\xi}$, à branchements finis, donc a une branche par le lemme de König ; mais ceci contredit la bonne fondation de $T_{\xi}$. On peut donc supposer qu'il existe $p$ tel que la suite $\left(s_{m}^{\prime}\lceil p)_{m}\right.$ soit constante, disons à $s$, et tel que la suite $\left(s_{m}^{\prime}(p)\right)_{m}$ tende vers l'infini. Alors par l'inégalité triangulaire on a que la distance de $\left(z_{m}, t_{m}\right)$ à $G\left(g_{s}\right)$, dans $Z_{0} \times T_{0}$, est au plus $2^{-s_{m}^{\prime}(p)}$, donc comme la convergence a lieu aussi dans $Z_{0} \times T_{0}$, on a $(z, t) \in{\overline{G\left(g_{s}\right)}}^{Z_{0} \times T_{0}} \cap(Z \times T)$. Mais comme avant, on en déduit que $(z, t)$ est dans $G\left(f_{s}\right) \subseteq G\left(f_{\emptyset}\right) \cup \bigcup_{s \in T_{\xi}^{\prime}} C_{s}$

On a donc que $\overline{B_{p}} \subseteq G\left(f_{\emptyset}\right) \cup \bigcup_{s \in T_{\xi}^{\prime}} C_{s}$. Si $|s|$ est paire, on montre que $C_{s} \subseteq \overline{B_{p}}$, ce qui montrera que $\overline{B_{p}}=G\left(f_{\emptyset}\right) \cup \bigcup_{s \in T_{\xi}^{\prime}} C_{s}=B_{p} \cup B_{i}$. Or on a

$$
C_{s}=\bigcup_{n \in \omega} G\left(f_{s \frown n}\right) \subseteq \bigcup_{n \in \omega} \overline{C_{s \frown n}} \subseteq \overline{\bigcup_{n \in \omega} C_{s \frown n}} \subseteq \overline{B_{p}}
$$

Ceci termine la preuve.

Par [Lo-SR], on a, pour $\xi<\omega_{1}$ impair, l'existence d'un compact $K_{\xi}$ et d'un vrai $D_{\xi}\left(\boldsymbol{\Sigma}_{1}^{0}\right)$ de $K_{\xi}$, disons $B_{\xi}$, tel que si $A$ est borélien d'un espace polonais $P, A$ n'est pas $\check{D}_{\xi}\left(\Sigma_{1}^{0}\right)$ de $P$ si et seulement s'il existe $f: K_{\xi} \rightarrow P$ injective continue telle que $f^{-1}(A)=B_{\xi}$. On a ici un analogue : si $A$ est borélien d'un produit d'espaces polonais, $A$ est non-pot $\left(\check{D}_{\xi}\left(\Sigma_{1}^{0}\right)\right)$ si et seulement s'il existe $B$ dans $D_{\xi}\left(\Sigma_{1}^{0}\right)\left\lceil\bar{B} \backslash \operatorname{pot}\left(\check{D}_{\xi}\left(\Sigma_{1}^{0}\right)\right)\right.$ et des injections continues $u$ et $v$ tels que $B=\bar{B} \cap(u \times v)^{-1}(A)$. Bien sûr, $B$ dépend ici de $A$, mais est toujours du même type $\left(B=D\left(\left(\bigcup_{f(s) \leq \eta} G\left(f_{s}\right)\right)_{\eta<\xi}\right)\right)$.

On va maintenant donner des versions du théorème 2.3 dans le cas où $A$ est à coupes verticales dénombrables, et dans le cas où $\xi=1$.

Lemme 2.4 (a) Soient $X$ et $Y$ des espaces polonais, $A$ (resp. B) un borélien de $X$ (resp. $Y$ ), et $f: A \rightarrow B$ countable-to-one borélienne. Alors il existe une partition de $A$ en ensembles boréliens, disons $\left(A_{n}\right)_{n \in \omega}$, telle que les restrictions de $f$ à $A_{n}$ soient injectives.

(b) Soient $X$ et $Y$ des espaces polonais, $A$ (resp. B) un presque-ouvert non vide de $X$ (resp. $Y$ ), et $g: B \rightarrow A$ surjective continue ouverte countable-to-one. Alors il existe un presque-ouvert non vide $A^{\prime}\left(\right.$ resp. $\left.B^{\prime}\right)$ de $X$ (resp. $\left.Y\right)$, contenu dans $A$ (resp. $\left.B\right)$, tel que la restriction de $g$ à $B^{\prime}$ soit un homéomorphisme de $B^{\prime}$ sur $A^{\prime}$.

Démonstration. (a) Par le théorème de Lusin, l'ensemble $\operatorname{Gr}(f)^{*}:=\{(y, x) /(x, y) \in \operatorname{Gr}(f)\}$ est la réunion dénombrable des graphes de fonctions boréliennes partielles, disons $f_{n}$, définies sur des boréliens. Puisque leur graphe est contenu dans $\operatorname{Gr}(f)^{*}$, les $f_{n}$ sont injectives, donc leurs images $C_{n}$ sont boréliennes. Il reste à poser $A_{n}:=C_{n} \backslash \bigcup_{p<n} C_{p}$.

(b) Soit $\left(B_{n}\right)$ une partition borélienne de $B$ donnée par le (a). Comme $B$ est non maigre relativement à $Y$, l'un des $B_{n}$ est non maigre. Ce dernier ayant la propriété de Baire s'écrit comme réunion disjointe d'un $G_{\delta}$ non maigre $G$ de $Y$ et d'une partie maigre $M$. Soit $U$ un ouvert non vide de $Y$ tel que $G \Delta U$ soit maigre, et posons $B^{\prime \prime}:=U \cap G$. Cet ensemble est un $G_{\delta}$ dense de $B \cap U$, qui est ouvert de $B$, donc $g\left[B^{\prime \prime}\right]$ est un analytique co-maigre de l'ouvert $g[B \cap U]$ de $A$. 
Soient donc $\left(U_{n}\right)$ une base de la topologie de $B^{\prime \prime}, V_{n}$ des ouverts de $g[B \cap U]$ tels que $g\left[U_{n}\right] \Delta V_{n}$ soit un maigre $M_{n}$ dans $g[B \cap U]$, et $A^{\prime}$ un $G_{\delta}$ dense de $g[B \cap U]$ contenu dans $g\left[B^{\prime \prime}\right] \backslash \bigcup_{n \in \omega} M_{n}$. Alors on peut poser $B^{\prime}:=B^{\prime \prime} \cap g^{-1}\left(A^{\prime}\right)$, comme on le vérifie facilement.

Théorème 2.5 Soient $X$ et $Y$ des espaces polonais, A un borélien à coupes verticales dénombrables de $X \times Y$, et $\xi$ un ordinal dénombrable non nul.

(a) Si $\xi$ est pair, A est non-pot $\left(D_{\xi}\left(\Sigma_{1}^{0}\right)\right)$ si et seulement s'il existe des espaces polonais parfaits $Z$ et $T$ de dimension 0 , des ouverts-fermés non vides $A_{s}$ et $B_{s}$ (l'un dans $Z$ et l'autre dans $T$, pour $s$ dans $T_{\xi}$, et dans cet ordre si $|s|$ est paire), des surjections continues ouvertes $f_{s}$ de $A_{s}$ sur $B_{s}$, et des injections continues $u$ et $v$ tels que si $C_{s}:=\bigcup_{n \in \omega} G\left(f_{s \frown n}\right)$, on ait

$$
G\left(f_{s}\right) \subseteq \overline{C_{s}} \backslash\left(\bigcup_{t \in T_{\xi}^{\prime} / \text { parité }(|t|)=\text { parité }(|s|)} C_{t}\right),
$$

et si $B:=\bigcup_{s \in T_{\xi} /|s| \text { paire }} G\left(f_{s}\right), B=(u \times v)^{-1}(A)$.

(b) Si $\xi$ est impair, A est non-pot $\left(\check{D}_{\xi}\left(\Sigma_{1}^{0}\right)\right)$ si et seulement s'il existe des espaces polonais $Z$ et $T$ parfaits de dimension 0 , des ouverts-fermés non vides $A_{s}$ et $B_{s}$ (l'un dans $Z$ et l'autre dans $T$, pour $s$ dans $T_{\xi}$, et dans cet ordre si $|s|$ est impaire), des surjections continues ouvertes $f_{s}$ de $A_{s}$ sur $B_{s}$, et des injections continues $u$ et $v$ tels que si $C_{s}:=\bigcup_{n \in \omega} G\left(f_{s} \frown_{n}\right)$, on ait

$$
G\left(f_{s}\right) \subseteq \overline{C_{s}} \backslash\left(\bigcup_{t \in T_{\xi}^{\prime} / \text { parité }(|t|)=\text { parité }(|s|)} C_{t}\right),
$$

et si $B:=\bigcup_{s \in T_{\xi} /|s| \text { impaire }} G\left(f_{s}\right)$, on ait $B=(u \times v)^{-1}(A)$.

Démonstration. Elle est identique à la preuve du théorème 2.3, à ceci près qu'on remplace la condition (ii) $\operatorname{par} \operatorname{Gr}\left(g_{s \frown n}\right)\left(\right.$ ou $\left.\operatorname{Gr}\left(g_{s \frown n}\right)^{*}\right) \subseteq \mathcal{B}\left[\left(x_{n}^{s}, g_{s}\left(x_{n}^{s}\right)\right), 2^{-\Sigma_{i \leq|s|}\left(s^{\frown} n(i)+1\right)}\right]$ si $s \in T_{\xi}^{\prime}$ et $f(s) \neq 1$, et $A \cap\left(Z_{0} \times T_{0}\right)=\bigcup_{n \in \omega} \operatorname{Gr}\left(g_{s \frown n}\right)$ si $f(s)=1$. Si $A$ est la $\Delta_{1}^{1}(\beta)$-réunion de $\left(h_{n}\right)$, on prend pour la suite $\left(\operatorname{Gr}\left(g_{s} \neg n\right)\right)_{n}$ la suite des traces de $\operatorname{Gr}\left(h_{n}\right)$ sur $Z_{0} \times T_{0}$ qui sont non vides (ceci si $f(s)=1$ ). Si $f(s)>0$ est pair, on raisonne comme dans la preuve de 2.3 pour construire $g_{s}$, à ceci près que si le graphe n'est pas contenu dans $Z_{0} \times T_{0}$ mais dans $T_{0} \times Z_{0}$, on applique le lemme 2.4 pour avoir le graphe dans le sens souhaité.

Théorème 2.6 Soient $X$ et $Y$ des espaces polonais, $A$ un borélien à coupes verticales dénombrables de $X \times Y$. Alors A est non-pot $\left(\mathbf{\Pi}_{1}^{0}\right)$ si et seulement s'il existe des espaces polonais $Z$ et $T$ parfaits de dimension 0 , une suite d'ouverts-fermés $\left(A_{n}\right)$ (resp. $\left.\left(B_{n}\right)\right)$ de $Z$ (resp. T), des surjections continues ouvertes $f_{n}$ de $A_{n}$ sur $B_{n}$, et des fonctions continues $u$ et $v$ tels que si $B=\bigcup_{n>0} G r\left(f_{n}\right)$, on ait $\emptyset \neq G r\left(f_{0}\right) \subseteq \bar{B} \backslash B$ et $B=(u \times v)^{-1}(A)$.

Démonstration. Elle est identique à celle du théorème 2.5 si $a_{\emptyset} \subseteq Z_{0}$. Dans le cas où $a_{\emptyset} \subseteq T_{0}$, on montre comme dans 2.5 que l'ensemble

$$
L:=G\left(g_{\emptyset}\right) \cap\left[\left(M_{0} \cap \Omega_{Z_{0}}^{\alpha} \cap D_{Z_{0}}^{\alpha}\right) \times\left(N_{0} \cap \Omega_{T_{0}}^{\alpha} \cap D_{T_{0}}^{\alpha}\right)\right] \cap \overline{A \cap\left(Z_{0} \times T_{0}\right)}{ }^{\Sigma_{Z_{0}}^{\alpha} \times \Sigma_{T_{0}}^{\alpha}}
$$

est non vide. 
On définit alors $A_{0}$ comme étant la projection de $L$ sur $T_{0}, B_{0}:=Z:=T:=A_{0} ; f_{0}$ est l'application identique. Soient $\left(h_{n}\right)_{n>0}$ comme dans la preuve de $2.5, E_{n}$ le domaine de $h_{n}, D_{0}$ la projection de $L$ sur $Z_{0}$, et $g_{0}: A_{0} \rightarrow D_{0}$ la restriction de $g_{\emptyset}$ à $A_{0}$. On définit $A_{n}:=g_{0}^{-1}\left(h_{n}^{-1}\left(A_{0}\right)\right)$, $B_{n}:=A_{0} \cap h_{n}\left[E_{n} \cap D_{0}\right]$, et $f_{n}$ comme étant la restriction de $h_{n} \circ g_{0}$ à $A_{n}$, si $n>0$. Enfin, on pose $u:=g_{0}, v(y)=y$ si $y \in A_{0}$. Vérifions que ces objets conviennent. Si $x \in A_{0},(x, x) \notin B$ sinon $\left(g_{0}(x), x\right) \in A \cap \operatorname{Gr}\left(g_{\emptyset}\right)^{*}$. Si $U$ est un ouvert de $A_{0}$ contenant $x, g_{0}[U] \times U$ est un ouvert de $\left(M_{0} \cap \Omega_{Z_{0}}^{\alpha} \cap D_{Z_{0}}^{\alpha}\right) \times\left(N_{0} \cap \Omega_{T_{0}}^{\alpha} \cap D_{T_{0}}^{\alpha}\right)$ contenant $\left(g_{0}(x), x\right)$, donc rencontre $A$ en un point $(z, y)$; $z=g_{\emptyset}(t)$, où $t \in U$, et $(t, y) \in U^{2} \cap B \neq \emptyset$. Donc $\Delta\left(A_{0}\right)=\operatorname{Gr}\left(f_{0}\right) \subseteq \bar{B} \backslash B$.

Enfin, $(x, y) \in B \Leftrightarrow \exists n y=h_{n}\left(g_{0}(x)\right) \Leftrightarrow \exists n\left(g_{0}(x), y\right) \in \operatorname{Gr}\left(h_{n}\right) \Leftrightarrow\left(g_{0}(x), y\right) \in A$.

Soit $C_{0}$ la classe des fonctions de la forme $(x, y) \mapsto(u(x), v(y))$ ou $(x, y) \mapsto(u(y), v(x))$, où les fonctions $u$ et $v$ sont boréliennes.

Théorème 2.7 Soient $X$ et $Y$ des espaces polonais, $A$ un borélien pot $\left(\boldsymbol{\Sigma}_{3}^{0}\right) \cap \operatorname{pot}\left(\boldsymbol{\Pi}_{3}^{0}\right)$ de $X \times Y$.

(a) A est non-pot $\left(\mathbf{\Pi}_{1}^{0}\right)$ si et seulement s'il existe des espaces polonais $Z^{\prime}$ et $T^{\prime}$ parfaits de dimension 0 , une suite d'ouverts-fermés $\left(A_{n}\right)$ (resp. $\left.\left(B_{n}\right)\right)$ de $Z^{\prime}$ (resp. $\left.T^{\prime}\right)$, des surjections continues ouvertes $f_{n}$ de $A_{n}$ sur $B_{n}$, et une fonction continue $f$ de $C_{0}$ tels que si $B=\bigcup_{n>0} G r\left(f_{n}\right)$, on ait

$$
\emptyset \neq G r\left(f_{0}\right)=\bar{B} \backslash B
$$

et $B=f^{-1}(A) \cap \bar{B}$.

(b) A est non-pot $\left(\mathbf{\Pi}_{1}^{0}\right)$ si et seulement s'il existe des espaces polonais $Z$ et $T$ parfaits de dimension 0 non vides, une suite d'ouverts denses $\left(E_{n}\right)$ de $Z$, des applications continues ouvertes $g_{n}$ de $E_{n}$ dans $T$, et une fonction continue $g$ de $C_{0}$ tels que $\left(g_{n}\right)_{n>0}$ converge uniformément vers $g_{0}$ sur $\bigcap_{n \in \omega} E_{n}$, $\operatorname{Gr}\left(g_{0}\right) \subseteq g^{-1}(\check{A})$ et $\bigcup_{n>0} \operatorname{Gr}\left(g_{n}\right) \subseteq g^{-1}(A)$.

Démonstration. (a) Dans le cas où $a_{\emptyset} \subseteq Z_{0}$, la preuve est identique à celle du théorème 2.3 jusqu'à la construction des $g_{s}$ comprise. Soient $I_{0}:=\left\{n / a_{(n)} \subseteq Z_{0}\right\}, I_{1}:=\left\{n / a_{(n)} \subseteq T_{0}\right\}$,

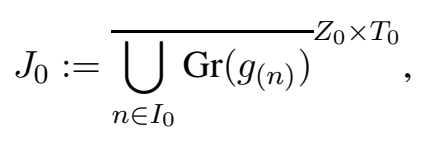

$J_{1}:={\overline{\bigcup_{n \in I_{1}} \operatorname{Gr}\left(g_{(n)}\right)^{*}}}^{Z_{0} \times T_{0}}$. Alors $\operatorname{Gr}\left(g_{\emptyset}\right) \subseteq J_{0} \cup J_{1}$, donc on trouve $i$ et un ouvert-fermé $U \times V$ de $Z_{0} \times T_{0}$ tels que $\emptyset \neq \operatorname{Gr}\left(g_{\emptyset}\right) \cap(U \times V) \subseteq J_{i}$. Supposons par exemple que $i=0$. Soit $g_{0}$ la restriction de $g_{\emptyset}$ à $U \cap g_{0}^{-1}(V)$, et $B^{\prime}:=\bigcup_{n \in I_{0}} \operatorname{Gr}\left(g_{(n)}\right)$; on a donc $\operatorname{Gr}\left(g_{0}\right) \subseteq{\overline{B^{\prime}}}^{Z_{0} \times T_{0}}$. On conclut de manière analogue à celle de 2.3 : on introduit $\alpha$, on montre que l'ensemble

$$
K:=\operatorname{Gr}\left(g_{0}\right) \cap(Z \times T) \cap \overline{B^{\prime} \cap[(Z \cap U) \times(T \cap V)]}{ }^{\Sigma_{Z_{0}}^{\alpha} \times \Sigma_{T_{0}}^{\alpha}}
$$

est non vide, ce qui implique que $I_{0}$ est infini ; on indexe alors $B^{\prime}$ par $\omega$. On définit $A_{0}$ et $B_{0}$ (resp. $A_{n}$ et $\left.B_{n}\right)$ comme étant les projections de $K$ (resp. $\left.\operatorname{Gr}\left(g_{(n)}\right) \cap[(Z \cap U) \times(T \cap V)]\right)$, et on pose $f_{0}:=g_{0}\left\lceil A_{0}, f_{n}:=g_{(n)}\left\lceil A_{n}\right.\right.$ si $n>0, Z^{\prime}:=Z \cap U, T^{\prime}:=T \cap V$, et $f$ est l'application identique. 
Si maintenant $i=1$, on montre comme dans 2.6 qu'on peut trouver deux fonctions continues $u$ et $v$, un espace polonais $Z^{\prime}$ parfait de dimension 0 , et des applications continues et ouvertes définies sur et d'images des ouverts-fermés de $Z^{\prime}, \varphi_{n}$, tels que si $A^{\prime}:=\bigcup_{n>0} \operatorname{Gr}\left(\varphi_{n}\right)$, on ait

$$
\Delta\left(Z^{\prime}\right) \subseteq(v \times u)^{-1}\left(\operatorname{Gr}\left(\varphi_{0}\right)\right),
$$

$\operatorname{Gr}\left(\varphi_{0}\right) \subseteq \operatorname{Gr}\left(g_{\emptyset}\right) \cap(U \times V), A^{\prime *} \subseteq(v \times u)^{-1}(A)$, et $\Delta\left(Z^{\prime}\right) \subseteq \overline{A^{\prime}} \backslash A^{\prime}$. Soit $\left(t_{n}\right)$ une suite dense de $Z^{\prime}$. Alors on construit des rectangles ouverts-fermés $W_{n}$ de $Z^{\prime 2}$ de diamètre au plus $2^{-n}$ et une suite strictement croissante $\left(m_{n}\right)$ telle que l'on ait $\left(t_{n}, t_{n}\right) \in W_{n}$,

$$
\emptyset \neq \operatorname{Gr}\left(\varphi_{m_{n}}\right) \cap W_{n} \subseteq{ }^{c}\left(\bigcup_{p<n} \operatorname{Gr}\left(\varphi_{m_{p}}\right)\right)
$$

Il est maintenant clair qu'on peut poser $A_{0}:=B_{0}:=T^{\prime}:=Z^{\prime}, f_{0}:=\operatorname{Id}_{A_{0}} ;$ si $n>0, A_{n}$ et $B_{n}$ sont les projections de $\operatorname{Gr}\left(\varphi_{m_{n}}\right) \cap W_{n}$, et $f_{n}$ est la restriction de $\varphi_{m_{n}}$ à $A_{n}$; enfin,

$$
f(x, y):=(v(y), u(x)) .
$$

Le cas où $a_{\emptyset} \subseteq T_{0}$ se traite de façon analogue.

(b) Supposons que $A$ est $\operatorname{pot}\left(\boldsymbol{\Pi}_{1}^{0}\right)$, en raisonnant par l'absurde. Alors si on pose $G:=\bigcap_{n \in \omega} E_{n}$, $\bigcup_{n \in \omega} \operatorname{Gr}\left(g_{n}\right) \cap(G \times T)$ est borélien à coupes fermées, donc on trouve un $G_{\delta}$ dense $H \subseteq G$ tel que $\bigcup_{n \in \omega} \operatorname{Gr}\left(g_{n}\right) \cap(H \times T)$ soit fermé dans $H \times T$. Par ailleurs,

$$
g^{-1}(A) \cap \bigcup_{n \in \omega} \operatorname{Gr}\left(g_{n}\right) \cap(H \times T)=\bigcup_{n>0} \operatorname{Gr}\left(g_{n}\right) \cap(H \times T)
$$

est pot $\left(\boldsymbol{\Pi}_{1}^{0}\right)$, donc on trouve un $G_{\delta}$ dense $K \subseteq T$ tel que les coupes de $E:=\bigcup_{n>0} \operatorname{Gr}\left(g_{n}\right) \cap(H \times K)$ soient fermées dans $K$. Alors $H \cap \bigcap_{n \in \omega} g_{n}^{-1}(K)$ est un $G_{\delta}$ dense de $Z$, et si $x$ est dans ce $G_{\delta}, g_{0}(x)$ est dans $\overline{E_{x}} \backslash E_{x}$, au sens de $K$, ce qui est la contradiction cherchée.

Inversement, soient $Z^{\prime}, T^{\prime}, A_{n}, B_{n}, f_{n}, f$, fournis par le (a). Posons, si $n>0$,

$$
H_{n}:=\left\{P \subseteq \boldsymbol{\Delta}_{1}^{0}\left[A_{0} \backslash\{\emptyset\} / \forall(U, V) \in P^{2}(U \neq V \Rightarrow U \cap V=\emptyset) \text { et } \exists p>0\left(U \subseteq A_{p} \text { et } \forall x \in U d\left(f_{0}(x), f_{p}(x)\right)<2^{-n}\right)\right\}\right. \text {. }
$$

Alors $H_{n}$ est non vide, puisqu'il contient $\emptyset$, et il est ordonné de manière inductive par l'inclusion, donc il admet un élément maximal $P_{n}:=\left\{U_{m}^{n} / m \in I_{n}\right\}$. Posons $E_{n}:=\bigcup_{m \in I_{n}} U_{m}^{n} ; E_{n}$ est dense dans $A_{0}$, par maximalité de $P_{n}$. On pose alors $Z:=A_{0}, T:=T^{\prime}, g:=f\left\lceil(Z \times T), E_{0}:=A_{0}\right.$, $g_{0}:=f_{0}$, et si $n>0, g_{n}(x):=f_{p_{m}}(x)$ si $x \in U_{m}^{n}$, où bien sûr $p_{m}$ est minimal tel que $U_{m}^{n} \subseteq A_{p_{m}}$ et $\forall x \in U_{m}^{n} d\left(f_{0}(x), f_{p_{m}}(x)\right)<2^{-n}$. Ces objets répondent clairement au problème.

Lemme 2.8 Soient $X$ et $Y$ des espaces polonais, $A$ un borélien à coupes verticales dénombrables de $X \times Y$, et $k$ un entier naturel non nul. Alors $\Gamma_{A} \neq D_{k}\left(\boldsymbol{\Sigma}_{1}^{0}\right)^{+}, \check{D}_{2 k}\left(\boldsymbol{\Sigma}_{1}^{0}\right)$, et $D_{2 k-1}\left(\boldsymbol{\Sigma}_{1}^{0}\right)\left(\Gamma_{A}\right.$ est la plus petite classe de Wadge $\Gamma$ telle que $A \in \operatorname{pot}(\Gamma)$, pour l'inclusion - cf [Lel]; $\Gamma^{+}$est le successeur de $\Gamma)$. 
Démonstration. Elle repose sur le fait que si $B$ est borélien à coupes verticales dénombrables et est $\operatorname{pot}\left(\boldsymbol{\Sigma}_{1}^{0}\right), B$ est en fait $\operatorname{pot}\left(\boldsymbol{\Delta}_{1}^{0}\right)$ (puisque sa projection est dénombrable).

Il suffit de montrer que $\Gamma_{A} \neq \check{D}_{2 k}\left(\boldsymbol{\Sigma}_{1}^{0}\right)$, la preuve de la troisième assertion étant analogue et celle de la première se déduisant des deux autres.

Supposons que $A=\check{U}_{2 k-1} \cup U_{0} \cup\left(U_{2} \backslash U_{1}\right) \cup \ldots \cup\left(U_{2 k-2} \backslash U_{2 k-3}\right)$; on a alors aussi

$$
A={ }^{c}\left(U_{2 k-1} \backslash U_{0}\right) \cup\left[\left(U_{2} \backslash U_{0}\right) \backslash\left(U_{1} \backslash U_{0}\right)\right] \cup \ldots \cup\left[\left(U_{2 k-2} \backslash U_{0}\right) \backslash\left(U_{2 k-3} \backslash U_{0}\right)\right] .
$$

Donc si on pose $V_{2 k-1}:=X \times Y$ et $V_{p}:=U_{p+1} \backslash U_{0}$ si $p<2 k-1$, on a

$$
A=\left(V_{1} \backslash V_{0}\right) \cup \ldots \cup\left(V_{2 k-1} \backslash V_{2 k-2}\right) .
$$

Ceci termine la preuve.

Corollaire 2.9 Soient $X$ et $Y$ des espaces polonais, A un borélien à coupes verticales dénombrables de $X \times Y$, et $k$ un entier naturel non nul. Alors on a les équivalences suivantes :

(a) A est non-pot $\left(\boldsymbol{\Delta}_{1}^{0}\right) \Leftrightarrow A$ est non-pot $\left(\boldsymbol{\Sigma}_{1}^{0}\right) \Leftrightarrow$ la projection de A sur $Y$ est non dénombrable.

(b) A est non-pot $\left(\check{D}_{2 k-1}\left(\boldsymbol{\Sigma}_{1}^{0}\right)\right) \Leftrightarrow A$ est non-pot $\left(\check{D}_{2 k}\left(\boldsymbol{\Sigma}_{1}^{0}\right)\right) \Leftrightarrow A$ est non-pot $\left(D_{2 k-1}\left(\boldsymbol{\Sigma}_{1}^{0}\right)^{+}\right)$.

(c) A est non-pot $\left(D_{2 k+1}\left(\Sigma_{1}^{0}\right)\right) \Leftrightarrow A$ est non-pot $\left(D_{2 k}\left(\Sigma_{1}^{0}\right)\right) \Leftrightarrow A$ est non-pot $\left(D_{2 k}\left(\Sigma_{1}^{0}\right)^{+}\right)$.

Ce résultat, couplé avec le théorème 2.5 , donne des caractérisations des ensembles potentiellement différences finies d'ouverts parmi les boréliens à coupes verticales dénombrables. Les classes $\{\emptyset\}, \boldsymbol{\Delta}_{1}^{0}, D_{2 n+2}\left(\boldsymbol{\Sigma}_{1}^{0}\right)$, et $\check{D}_{2 n+1}\left(\boldsymbol{\Sigma}_{1}^{0}\right)$ sont les seules de la forme $\Gamma_{A}$, avec $A$ borélien à coupes verticales dénombrables potentiellement différences finies d'ouverts, comme on le voit avec les résultats précédents et les ensembles suivants :

$$
\begin{aligned}
& A_{2 n+2}:=\left\{(\alpha, \beta) \in 2^{\omega} \times 2^{\omega} / \text { le nombre d'entiers où } \alpha \text { et } \beta \text { diffèrent est impair } \leq 2 n+1\right\}, \\
& A_{2 n+1}:=\left\{(\alpha, \beta) \in 2^{\omega} \times 2^{\omega} / \text { le nombre d'entiers où } \alpha \text { et } \beta \text { diffèrent est pair } \leq 2 n\right\} .
\end{aligned}
$$

Vérifions-le pour $A_{2 n+2}$, par exemple. Il est clairement $D_{2 n+2}\left(\Sigma_{1}^{0}\right)$, puisque si on pose

$$
U_{p}:=\left\{(\alpha, \beta) \in 2^{\omega} \times 2^{\omega} / \exists s \in \omega^{2 n+2-p} i \neq j \Rightarrow s(i) \neq s(j) \text { et } \forall i<|s| \alpha(s(i)) \neq \beta(s(i))\right\},
$$

$A_{2 n+2}=\bigcup_{p<2 n+2, p}$ impair $U_{p} \backslash U_{p-1}$; pour voir qu'il est non-pot $\left(\check{D}_{2 n+2}\left(\Sigma_{1}^{0}\right)\right)$, il suffit par le corollaire 2.9 de voir qu'il est non-pot $\left(\check{D}_{2 n+1}\left(\Sigma_{1}^{0}\right)\right)$. On va pour ce faire appliquer le théorème 2.5. On pose $f_{\emptyset}(\alpha)=\alpha$, et si $|t| \leq 2 n, f_{t-m}(\alpha)(p)=f_{t}(\alpha)(p) \Leftrightarrow p \neq m$. Posons également $\phi_{m}(\alpha)(p)=\alpha(p) \Leftrightarrow p \neq m$. Alors $\phi_{m}$ est un homéomorphisme, et comme $f_{t-m}=\phi_{m} \circ f_{t}$, on a par récurrence que les $f_{t}$ sont des homéomorphismes de $2^{\omega}$ sur lui-même. Par récurrence sur $|t| \leq 2 n+1$, on voit que le nombre de $p$ tels que $\alpha(p) \neq f_{t}(\alpha)(p)$ a même parité que $|t|$ et est $\leq|t|$. On en déduit que $\bigcup_{s \in \omega \leq 2 n+1} /|s|$ paire $C_{s} \subseteq A_{2 n+2}$. Enfin, si $\alpha \neq \beta$ en $n_{0}<n_{1}<\ldots<n_{2 p}$, avec $p \leq n$, et si on pose $\alpha_{0}=\alpha, \alpha_{l+1}=\phi_{n_{l}}\left(\alpha_{l}\right)$ si $l \leq 2 p, \alpha_{2 p+1}=\beta$, et $s=\left(n_{0}, \ldots, n_{2 p}\right)$, on vérifie que si $q \leq 2 p+1, \alpha_{q}=f_{s\lceil q}(\alpha)$; d'où $(\alpha, \beta) \in C_{s\lceil 2 p}$. 
Dans [SR], il est démontré le résultat suivant, dû à Hurewicz :

Théorème 2.10 Soit $X$ un espace polonais, et $A$ un borélien de $X$. Alors A est non- $\Pi_{2}^{0}$ si et seulement s'il existe $E$ dénombrable sans point isolé tel que $\bar{E} \backslash E \approx \omega^{\omega}$ et $E=A \cap \bar{E}$.

Théorème 2.11 Soient $X$ et $Y$ des espaces polonais, $A$ un borélien de $X \times Y$ à coupes verticales dénombrables. Alors $A$ est non-pot $\left(\Pi_{2}^{0}\right)$ si et seulement s'il existe des espaces polonais $Z$ et $T$ parfaits de dimension 0 non vides, des injections continues $u$ et $v$, une suite $\left(A_{n}\right)$ d'ouverts denses de $Z$, une suite $\left(f_{n}\right)$ d'applications continues et ouvertes de $A_{n}$ dans $T$, tels que pour tout $x$ dans $\bigcap_{n \in \omega} A_{n}$, l'ensemble $E_{x}:=\left\{f_{n}(x) / n \in \omega\right\}$ soit sans point isolé, $\overline{E_{x}} \backslash E_{x} \approx \omega^{\omega}$, et aussi $E_{x}=(u \times v)^{-1}(A)_{x} \cap \overline{E_{x}}$.

Démonstration. Supposons que $A$ soit $\operatorname{pot}\left(\Pi_{2}^{0}\right)$, en raisonnant par l'absurde. Alors il existe un $G_{\delta}$ dense $K$ de $T$ tel que les coupes verticales de $(Z \times K) \cap(u \times v)^{-1}(A)$ soient $G_{\delta}$ dans $K$, donc dans $T$. Par ailleurs, $\bigcap_{n \in \omega} A_{n} \cap \bigcap_{n \in \omega} f_{n}^{-1}(K)$ est $G_{\delta}$ dense de $Z$, puisque les fonctions $f_{n}$ sont continues et ouvertes. Donc on trouve $x$ dans ce $G_{\delta}$, et $E_{x}$ est polonais, puisque c'est une coupe verticale de $(Z \times K) \cap(u \times v)^{-1}(A)$ intersectée avec $\overline{E_{x}}$. De plus $E_{x}$ est dénombrable, non vide et sans point isolé, ce qui est contradictoire.

Inversement, on peut supposer, pour simplifier l'écriture, que $X$ et $Y$ sont récursivement présentés, et que $A$ est $\Delta_{1}^{1}$-réunion de graphes $\Delta_{1}^{1}$. Désignons par $W^{X}$ un ensemble $\Pi_{1}^{1} \subseteq \omega$ de codes pour les $\Delta_{1}^{1}$ de $X$, et par $C^{X} \subseteq \omega \times X$ un ensemble $\Pi_{1}^{1}$ dont les sections aux points de $W^{X}$ décrivent les $\Delta_{1}^{1}$ de $X$, et tel que la relation $\left(n \in W^{X}\right.$ et $(n, x) \notin C^{X}$ ) soit $\Pi_{1}^{1}$ (cf [Lo1]). Soit également $W \subseteq W^{X \times Y}$ un ensemble $\Pi_{1}^{1}$ de codes pour les $\Delta_{1}^{1} \cap \operatorname{pot}\left(\boldsymbol{\Sigma}_{2}^{0}\right)$ de $X \times Y$ (dont l'existence est démontrée dans [Lo2]). Posons

$$
H:=\cup\left\{(E \times F) \backslash A / E, F \in \Delta_{1}^{1} \text { et }(E \times F) \backslash A \in \operatorname{pot}\left(\Sigma_{2}^{0}\right)\right\} .
$$

On a

$$
\begin{aligned}
H(x, y) \Leftrightarrow & \exists n \in W \exists m:(m)_{0} \in W^{X} \text { et }(m)_{1} \in W^{Y} \text { et } \forall z \forall t \\
& {\left[\left(n \in W^{X \times Y} \text { et }(n, z, t) \notin C^{X \times Y}\right)\right. \text { ou }} \\
& \left.\left\{\left((m)_{0}, z\right) \in C^{X} \text { et }\left((m)_{1}, t\right) \in C^{Y} \text { et }(z, t) \notin A\right\}\right] \text { et } \\
& {\left[\left((m)_{0} \in W^{X} \text { et }\left((m)_{0}, z\right) \notin C^{X}\right) \text { ou }\left((m)_{1} \in W^{Y} \text { et }\left((m)_{1}, t\right) \notin C^{Y}\right)\right. \text { ou }} \\
& \left.(z, t) \in A \text { ou }(n, z, t) \in C^{X \times Y}\right] \\
& \text { et }\left((m)_{0}, x\right) \in C^{X} \text { et }\left((m)_{1}, y\right) \in C^{Y} \text { et }(x, y) \notin A .
\end{aligned}
$$

Donc $H$ est $\Pi_{1}^{1}$. Posons $N:=\check{A} \cap \check{H} ; N$ est $\Sigma_{1}^{1}$ et $G_{\delta}$ de $X \times Y$ muni de la topologie $\Delta_{X} \times \Delta_{Y}$ (cf [Lo2]). Posons $D_{X}:=\left\{x \in X / x \notin \Delta_{1}^{1}\right\}, \Omega_{X}:=\left\{x \in X / \omega_{1}^{x}=\omega_{1}^{\text {CK }}\right\}$, et $Z_{0}:=\Omega_{X} \cap D_{X}$, $T:=\Omega_{Y} \cap D_{Y}$. On munit $Z_{0}$ (resp. $T$ ) de la restriction de la topologie de Gandy-Harrington sur $X$ (resp. $Y$ ), de sorte que $Z_{0}$ et $T$ sont polonais parfaits de dimension 0 . On montre maintenant la propriété qui sera la clef de la construction à venir :

Si $U$ (resp. $V$ ) est $\Sigma_{1}^{1}$ et inclus dans $Z_{0}$ (resp. $T$ ), et si $N \cap(U \times V) \neq \emptyset$, alors l'ensemble $A \cap \overline{N \cap(U \times V)}^{Z_{0} \times T}$ est un sous- $\Sigma_{1}^{1}$ non vide de $U \times V$. 
En effet, $U$ (resp. $V$ ) est ouvert-fermé de $Z_{0}$ (resp. $T$ ), donc $\overline{N \cap(U \times V)}^{Z_{0} \times T} \subseteq U \times V ; A$, $Z_{0}, T, N, U$, et $V$ sont $\Sigma_{1}^{1}$, donc $A \cap \overline{N \cap(U \times V)}^{Z_{0} \times T}$ aussi, l'adhérence d'un $\Sigma_{1}^{1}$ pour le produit des topologies de Gandy-Harrington restant $\Sigma_{1}^{1}$, par double application du théorème de séparation. Posons $O:=N \cap(U \times V)$. Supposons que $A \cap \bar{O}^{\Sigma_{X} \times \Sigma_{Y}}=\emptyset$. Alors, par double application du théorème de séparation, $A \cap \bar{O}^{\Delta_{X} \times \Delta_{Y}}=\emptyset$, donc on a la triple inclusion

$$
(U \times V) \backslash A \subseteq O \cup H \subseteq \bar{O}^{\Delta_{X} \times \Delta_{Y}} \cup H \subseteq \check{A} .
$$

Donc $(U \times V) \backslash A$ et $A$ sont deux $\Sigma_{1}^{1}$ séparables par un $\operatorname{pot}\left(\Sigma_{2}^{0}\right)$; ils peuvent par conséquent être séparés par un ensemble $K \in \Delta_{1}^{1} \cap \operatorname{pot}\left(\boldsymbol{\Sigma}_{2}^{0}\right)$ (cf [Lo2]). On a $U \times V \subseteq K \cup A$, donc on peut trouver $\mathcal{U}$ et $\mathcal{V}$, deux $\Delta_{1}^{1}$ tels que $U \times V \subseteq \mathcal{U} \times \mathcal{V} \subseteq K \cup A$. On a donc $(\mathcal{U} \times \mathcal{V}) \backslash A \subseteq H$, puis $O \subseteq H \backslash H=\emptyset$, ce qui est absurde. On a donc que $A \cap \bar{O}^{\Sigma_{X} \times \Sigma_{Y}} \neq \emptyset$. Or $O \subseteq D_{X} \times D_{Y}$, donc $\bar{O}^{\Sigma_{X} \times \Sigma_{Y}} \subseteq D_{X} \times D_{Y}$. Donc $\left(D_{X} \times D_{Y}\right) \cap A \cap \bar{O}^{\Sigma_{X} \times \Sigma_{Y}} \neq \emptyset$ et est $\Sigma_{1}^{1}$, donc rencontre $\Omega_{X \times Y} \subseteq \Omega_{X} \times \Omega_{Y}$, donc $\left(Z_{0} \times T\right) \cap A \cap \bar{O}^{\Sigma_{X} \times \Sigma_{Y}} \neq \emptyset$.

Soit $d_{1}$ (resp. $d_{2}$ ) une distance $\leq 1 / 2$ qui rende $Z_{0}$ (resp. $T$ ) complet, et $d$ la distance sur $Z \times T$ définie par $d((x, y),(z, t)):=d_{1}(x, z)+d_{2}(y, t)$. Soit $d^{\prime}$ une distance $\leq 1$ sur $N \cap\left(Z_{0} \times T\right)$, qui le rende complet (c'est un $G_{\delta}$ de $Z_{0} \times T$, donc un espace polonais). On vérifie sans peine que si on pose $d_{x}^{\prime}(y, t):=d^{\prime}((x, y),(x, t))$, alors $\left(N_{x} \cap T, d_{x}^{\prime}\right)$ est métrique complet. On pose, si $s$ est une suite finie d'entiers non nuls, $\nu(s):=\Sigma_{i<|s|} s(i)(\nu(\emptyset)=0)$.

On construit, par récurrence sur $|s|$, des ouverts non vides de $Z_{0}, \mathcal{A}_{s}$, des $G_{\delta}$ denses $\mathcal{G}_{s}$ de $\mathcal{A}_{s}$, des applications continues et ouvertes $g_{s}: \mathcal{A}_{s} \rightarrow T$, des ouverts à coupes verticales ouvertes-fermées de $Z_{0} \times T, \omega_{s}$, et des ouverts de $N \cap\left(Z_{0} \times T\right), G_{s}$, tels que

$$
\begin{aligned}
& \text { (a) } \operatorname{Gr}\left(g_{s}\right) \subseteq A \cap \omega_{s} \cap{\overline{G_{s}}}^{Z_{0} \times T} \\
& \text { (b) } \omega_{s \frown n} \subseteq \omega_{s}, \omega_{s \frown n} \cap \omega_{s \frown m}=\emptyset \text { si } n \neq m \\
& \text { (c) } \forall x \in Z_{0} \overline{G_{s}^{x} n} T^{T} \cap N_{x} \subseteq G_{s}^{x} \subseteq \omega_{s}^{x} \\
& \text { (d) } \mathcal{A}_{s} \Delta \mathcal{A}_{s \frown n} \text { est maigre et } \forall x \in \mathcal{A}_{s \frown n} \cap \mathcal{G}_{s} \quad d_{2}\left(g_{s}(x), g_{s \frown n}(x)\right) \leq 2^{1-\nu(s \frown n)} \\
& \text { (e) } \forall x \in Z_{0} \quad \delta_{2}\left(\omega_{s}^{x}\right) \leq 2^{-\nu(s)} \\
& \text { (f) } \forall x \in Z_{0} \quad \delta_{x}^{\prime}\left(G_{s}^{x}\right) \leq 2^{-|s|}
\end{aligned}
$$

On pose $\omega_{\emptyset}:=Z_{0} \times T, G_{\emptyset}:=\left(Z_{0} \times T\right) \cap N$. L'ensemble $G_{\emptyset}$ est non vide. En effet, $N$ rencontre $D_{X} \times D_{Y}$, sinon $N$ serait $\operatorname{pot}\left(\boldsymbol{\Delta}_{1}^{0}\right)$ et $A \cup H$ aussi, donc $A=(A \cup H) \cap \check{H}$ serait $\operatorname{pot}\left(\boldsymbol{\Pi}_{2}^{0}\right)$, ce qui est exclus. Donc $N \cap\left(D_{X} \times D_{Y}\right)$, qui est $\Sigma_{1}^{1}$, rencontre $\Omega_{X \times Y} \subseteq \Omega_{X} \times \Omega_{Y}$ et $G_{\emptyset} \neq \emptyset$. Par la propriété-clef, $A \cap{\overline{G_{\emptyset}}}^{Z} \times T$ est un sous- $\Sigma_{1}^{1}$ non vide de $\omega_{\emptyset}$, donc $A \cap \omega_{\emptyset} \cap{\overline{G_{\emptyset}}} Z_{0} \times T$ est réunion de graphes $\Sigma_{1}^{1}$ dont l'un, disons $\operatorname{Gr}\left(g_{\emptyset}\right)$, est non vide. Il reste à appeler $\mathcal{A}_{\emptyset}$ le domaine de $g_{\emptyset}$ pour terminer la construction au premier cran. Admettons donc avoir construit $\mathcal{A}_{s}, g_{s}, \omega_{s}, G_{s}$ pour $|s| \leq p$ et $\mathcal{G}_{s}$ pour $|s|<p$ vérifiant (a)-(f), et soit $s$ de longueur $p$. 
On commence par construire, par récurrence sur $n$, les $\omega_{s}{ }_{n}$, une suite décroissante $\left(E_{n}\right)$ de $G_{\delta}$ denses de $\mathcal{A}_{s}$, des applications continues $h_{n}: E_{n} \rightarrow T$, et des ouverts $V_{n}$ de $Z_{0} \times T$ tels que

$$
\begin{aligned}
& \text { (1) } \operatorname{Gr}\left(h_{n}\right) \subseteq \omega_{s \frown n} \cap G_{s} \\
& \text { (2) } \omega_{s \frown n} \subseteq \omega_{s} \text { et } \omega_{s \frown n} \cap \omega_{s \frown m}=\emptyset \text { si } n \neq m \\
& \text { (3) } \forall x \in E_{n} \quad d_{2}\left(h_{n}(x), g_{s}(x)\right)<2^{-\nu(s \frown n)} \\
& \text { (4) } \forall x \in Z_{0} \quad \delta_{2}\left(\omega_{s \frown n}^{x}\right)<2^{-\nu(s \frown n)} \\
& \text { (5) } \operatorname{Gr}\left(g_{s}\left\lceil E_{n}\right) \subseteq V_{n} \subseteq{ }^{c}\left(\bigcup_{1 \leq m \leq n} \omega_{s \frown m}\right)\right.
\end{aligned}
$$

Admettons avoir construit ces objets pour $1 \leq m \leq n$, ce qui est fait pour $n=0$. Soit $x$ un élément de $\mathcal{A}_{s}$. Comme $g_{s}$ est continue en $x$, on trouve un voisinage ouvert $U_{x}$ de $x$ contenu dans $\mathcal{A}_{s}$ tel que si $z \in U_{x}, d_{2}\left(g_{s}(x), g_{s}(z)\right)<\varepsilon:=2^{-\nu\left(s^{\frown}(n+1)\right)-1}$. De telle façon que si $(z, t) \in U_{x} \times \mathcal{B}\left(g_{s}(x), \varepsilon\right)$, on a $d_{2}\left(t, g_{s}(z)\right)<2^{-\nu\left(s^{\frown}(n+1)\right)}$. Posons $\mathcal{U}_{n+1}:=V_{n} \cap \bigcup_{x \in \mathcal{A}_{s}} U_{x} \times \mathcal{B}\left(g_{s}(x), \varepsilon\right)$, et soit $O_{n}$ un ouvert dense de $\mathcal{A}_{s}$ contenant $E_{n}$ tel que $\operatorname{Gr}\left(g_{s}\right) \cap V_{n}=\operatorname{Gr}\left(g_{s}\left\lceil O_{n}\right)\right.$. Soit $k_{n+1}$ une fonction Baire-mesurable uniformisant $\mathcal{U}_{n+1} \cap \omega_{s} \cap G_{s}$ sur sa projection $\Pi$ qui est un ouvert de $Z_{0}$, et soit $F_{n+1}$ un $G_{\delta}$ dense de $\Pi$ sur lequel la restriction de $k_{n+1}$ est continue. Alors $O_{n} \backslash F_{n+1}$ est maigre car $O_{n} \cap \Pi$ est ouvert dense de $O_{n}$; en effet, si $U$ est ouvert non vide de $O_{n}$ et $x \in U,\left(x, g_{s}(x)\right) \in(U \times T) \cap \omega_{s} \cap \mathcal{U}_{n+1}$. Donc, comme $\operatorname{Gr}\left(g_{s}\right) \subseteq{\overline{G_{s}}}^{Z_{0} \times T}$, on trouve $(z, t)$ dans $G_{s} \cap(U \times T) \cap \omega_{s} \cap \mathcal{U}_{n+1}$, et $z \in U \cap \Pi$. De tout ceci résulte que $\mathcal{A}_{s} \backslash F_{n+1}$ est maigre, donc $E_{n+1}:=F_{n+1} \cap E_{n}$ est $G_{\delta}$ dense de $\mathcal{A}_{s}$ et $h_{n+1}:=k_{n+1}\left\lceil E_{n+1}\right.$ est continue. Si $x \in E_{n+1},\left(x, h_{n+1}(x)\right) \in \mathcal{U}_{n+1}$, donc

$$
d_{2}\left(h_{n+1}(x), g_{s}(x)\right)<2^{-\nu\left(s^{\frown}(n+1)\right)} .
$$

Dans $E_{n+1} \times T, \operatorname{Gr}\left(h_{n+1}\right)$ et $\operatorname{Gr}\left(g_{s}\left\lceil E_{n+1}\right)\right.$ sont deux fermés disjoints, donc séparables par un ouvertfermé $\theta$. Par la propriété de réduction des ouverts, on trouve donc 2 ouverts disjoints de $Z_{0} \times T$, $\mathcal{T}$ et $\mathcal{W}$ tels que $\theta=\mathcal{T} \cap\left(E_{n+1} \times T\right)$, et $\left(E_{n+1} \times T\right) \backslash \theta=\mathcal{W} \cap\left(E_{n+1} \times T\right)$. Comme $\operatorname{Gr}\left(h_{n+1}\right) \subseteq \mathcal{T}$, on trouve pour chaque $x$ dans $E_{n+1}$ un rectangle ouvert-fermé $V_{x} \times W_{x}$ tel que

$$
\left(x, h_{n+1}(x)\right) \in V_{x} \times W_{x} \subseteq \mathcal{T} \cap \omega_{s} \cap V_{n},
$$

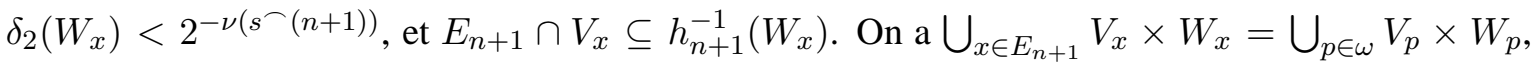
par Lindelöf. Réduisons la suite $\left(V_{p}\right)$ en $\left(V_{p}^{\prime}\right)$; on peut alors poser $\omega_{s-(n+1)}:=\bigcup_{p \in \omega} V_{p}^{\prime} \times W_{p}$ et $V_{n+1}:=V_{n} \cap \mathcal{W}$, comme on le vérifie facilement.

Revenons à la construction principale ; on a déjà assuré (b) et (e). Si $x \in E_{n}$, on peut trouver un ouvert-fermé de $N, \mathcal{U}_{x}:=\left(\mathcal{V}_{x} \times \mathcal{W}_{x}\right) \cap N$, de diamètre au plus $2^{-|s|-1}$ pour $d^{\prime}$, vérifiant

$$
\mathcal{V}_{x} \cap E_{n} \subseteq h_{n}^{-1}\left(\mathcal{W}_{x}\right),
$$

et si $Z_{s}$ est ouvert de $Z_{0} \times T$ tel que $G_{s}=Z_{s} \cap N$, on ait

$$
\left(x, h_{n}(x)\right) \in \mathcal{V}_{x} \times \mathcal{W}_{x} \subseteq \omega_{s} \neg n \cap Z_{s} \cap\left(\mathcal{A}_{s} \times T\right) .
$$

On a donc que $\operatorname{Gr}\left(h_{n}\right) \subseteq \bigcup_{x \in E_{n}} \mathcal{U}_{x}=\bigcup_{m \in \omega} \mathcal{U}_{m}$, par Lindelöf. On réduit la suite $\left(\mathcal{V}_{m}\right)$ en $\left(\mathcal{V}_{m}^{\prime}\right)$, et on pose $G_{s \frown n}:=\bigcup_{m \in \omega}\left(\mathcal{V}_{m}^{\prime} \times \mathcal{W}_{m}\right) \cap N$, et les conditions (c) et (f) sont réalisées, puisque $G_{s \frown n}^{x}$ est vide ou l'un des $\mathcal{W}_{m} \cap N_{x}$, donc est ouvert-fermé de $N_{x}$. De plus, on a $G_{s} \frown n \supseteq \operatorname{Gr}\left(h_{n}\right)$. 
A l'aide d'une nouvelle numérotation, on peut écrire $G_{s \frown n}=\bigcup_{p \in \omega}\left(L_{p} \times M_{p}\right) \cap N, L_{p}$ et $M_{p}$ étant deux $\Sigma_{1}^{1}$. Posons $E_{n, p}:=A \cap{\overline{N \cap\left(L_{p} \times M_{p}\right)}}^{Z_{0} \times T} \cap \omega_{s} \frown n ; E_{n, p}$ est réunion de graphes $\Sigma_{1}^{1}$. On peut donc trouver une suite de graphes $\Sigma_{1}^{1}$, contenus dans $E_{n, p}$, dont les domaines sont deux à deux disjoints, et tels que la réunion de ces domaines, disons $O_{n, p}$ forme un ouvert tel que $\bigcup_{q \leq p} O_{n, q}$ soit dense dans $\bigcup_{q \leq p} \Pi_{X}^{\prime \prime} E_{n, p}$ (une telle construction est possible avec le lemme de Zorn, par exemple). On fait ceci par récurrence sur $p$, de sorte que $\mathcal{A}_{s\urcorner n}:=\bigcup_{p \in \omega} O_{n, p}$ est un ouvert dense de $\bigcup_{p \in \omega} \Pi_{X}^{\prime \prime} E_{n, p}$. On appelle $g_{s} \frown n$ le recollement des fonctions définies sur les $O_{n, p}$, et la condition (a) est satisfaite, ainsi que la seconde partie de la condition (d), si on pose $\mathcal{G}_{s}:=\bigcap_{n \geq 1} E_{n}$, par (4). Reste à voir que $\mathcal{A}_{s} \Delta \mathcal{A}_{s} \frown n$ est maigre pour clore la construction. Quitte à remplacer $\mathcal{A}_{s} \frown n$ par $\mathcal{A}_{s} \cap \mathcal{A}_{s \frown n}$, il suffit de voir que $\mathcal{A}_{s} \backslash \mathcal{A}_{s \frown n}$ est maigre. Posons $\Pi_{p}:=\Pi_{X}^{\prime \prime}\left(L_{p} \times M_{p}\right) \cap N$ et $\Pi_{p}^{\prime}:=\Pi_{X}^{\prime \prime} E_{n, p}$. On a

$$
\begin{aligned}
\mathcal{A}_{s} \backslash \mathcal{A}_{s \frown n} & \subseteq \mathcal{A}_{s} \backslash\left(\bigcup_{p \in \omega} \Pi_{p}^{\prime}\right) \cup \bigcup_{p \in \omega} \Pi_{p}^{\prime} \backslash \mathcal{A}_{s \frown n} \\
& \subseteq \mathcal{A}_{s} \backslash E_{n} \cup E_{n} \backslash\left(\bigcup_{p \in \omega} \Pi_{p}^{\prime}\right) \cup \bigcup_{p \in \omega} \Pi_{p}^{\prime} \backslash \mathcal{A}_{s \frown n} \\
& \subseteq \mathcal{A}_{s} \backslash E_{n} \cup \bigcup_{p \in \omega} \Pi_{p} \backslash \Pi_{p}^{\prime} \cup \bigcup_{p \in \omega} \Pi_{p}^{\prime} \backslash \mathcal{A}_{s \frown n} .
\end{aligned}
$$

Il suffit donc de voir que $\Pi_{p} \cap \Pi_{p}^{\prime}$ est ouvert dense de $\Pi_{p}$; mais ceci est une conséquence facile de la propriété clef.

On définit maintenant les objets recherchés : $Z:=\mathcal{A}_{\emptyset}$, $u$ et $v$ sont les injections canoniques. Soit $\varphi: \omega \rightarrow(\omega \backslash\{0\})^{<\omega} \times \omega$ bijective, et $\left(U_{m}^{s}\right)_{m}$ une suite d'ouverts denses de $\mathcal{A}_{s}$ telle que $\mathcal{G}_{s}=\bigcap_{m \in \omega} U_{m}^{s}$. On pose $A_{n}:=\mathcal{A}_{\emptyset} \cap U_{\varphi_{1}(n)}^{\varphi_{0}(n)}$ et $f_{n}:=g_{\varphi_{0}(n)}\left\lceil A_{n}\right.$. L'ouvert $A_{n}$ est dense dans $Z$ par la première partie de (d), et $E_{x}$ est sans point isolé par la seconde partie de (d) (on a l'égalité $\left.\bigcap_{n \in \omega} A_{n}=\bigcap_{s \in(\omega \backslash\{0\})<\omega} \mathcal{G}_{s}\right)$.

La fin de la preuve est semblable à la preuve du théorème d'Hurewicz dans [SR]. Posons, si $x \in \bigcap_{n \in \omega} A_{n}, M_{k}:=\left\{g_{s}(x) /|s| \leq k\right\}$. Alors $M_{k}$ est fermé dans $T$, par récurrence sur k : si $M_{k}^{\varepsilon}:=\left\{y \in T / d_{2}\left(y, M_{k}\right) \leq \varepsilon\right\}$, on a $M_{k+1}=\bigcap_{\varepsilon>0}\left[M_{k}^{\varepsilon} \cup\left(M_{k+1} \backslash M_{k}^{\varepsilon}\right)\right]$, et $M_{k+1} \backslash M_{k}^{\varepsilon}$ est fini, $\operatorname{par}(\mathrm{d})$.

Si $k \in \omega$ et $y \in \overline{E_{x}} \backslash E_{x}, y \notin M_{k}$, donc il existe $\varepsilon>0$ tel que $y \notin M_{k}^{\varepsilon}$. Si $s \in(\omega \backslash\{0\})^{k}$ et $(x, t) \in \omega_{s}, d_{2}\left(t, M_{k}\right) \leq d_{2}\left(t, g_{s}(x)\right) \leq \delta_{2}\left(\omega_{s}^{x}\right) \leq 2^{-\nu(s)} \leq \varepsilon$ dès que $\nu(s) \geq k_{0}$, donc $\omega_{s}^{x} \subseteq M_{k}^{\varepsilon}$, sauf pour un nombre fini de $s$ dans $(\omega \backslash\{0\})^{k}$. Donc si $\mathcal{H}:=\left\{s \in(\omega \backslash\{0\})^{k} / \omega_{s}^{x} \nsubseteq M_{k}^{\varepsilon}\right\}$, on a $E_{x}=M_{k} \cup\left\{g_{s}(x) /|s|>k\right\} \subseteq M_{k} \cup \bigcup_{|t|>k} \omega_{t}^{x} \subseteq M_{k} \cup \bigcup_{|s|=k} \omega_{s}^{x} \subseteq M_{k}^{\varepsilon} \cup \bigcup_{s \in \mathcal{H}} \omega_{s}^{x}$ et $\overline{E_{x}} \subseteq M_{k}^{\varepsilon} \cup \bigcup_{s \in \mathcal{H}} \omega_{s}^{x} \subseteq M_{k}^{\varepsilon} \cup \bigcup_{|s|=k} \omega_{s}^{x}$. Donc on trouve une unique suite $\sigma$ dans $(\omega \backslash\{0\})^{\omega}$ telle que $y \in \bigcap_{s \prec \sigma} \omega_{s}^{x}$. La suite décroissante de fermés non vides dont les diamètres tendent vers 0 de

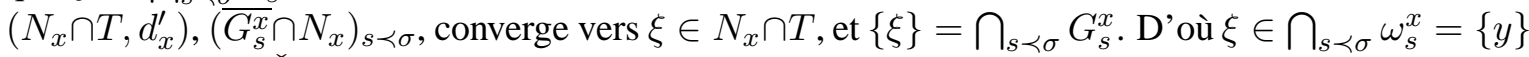
et $(x, y) \in N \subseteq \check{A}$. Posons

$$
h: \begin{cases}(\omega \backslash\{0\})^{\omega} & \rightarrow T \\ \sigma & \mapsto \bigcap_{s \prec \sigma} \overline{G_{s}^{x}}\end{cases}
$$

La fonction $h$ est bien définie, à valeurs dans $N_{x} \subseteq \check{E}_{x}$. Si $\sigma \in(\omega \backslash\{0\})^{\omega}, h(\sigma) \in \bigcap_{s \prec \sigma} \omega_{s}^{x}$ et $\omega_{s}^{x} \cap E_{x} \neq \emptyset$; par conséquent, $h$ est à valeurs dans $\overline{E_{x}}$. On a vu que $\overline{E_{x}} \backslash E_{x} \subseteq h^{\prime \prime}(\omega \backslash\{0\})^{\omega}$. Par (b), $h$ est injective, et par (e), $h$ est continue. Comme $h^{\prime \prime} N_{s}=\omega_{s}^{x} \cap\left(\overline{E_{x}} \backslash E_{x}\right), h$ est bien un homéomorphisme de $(\omega \backslash\{0\})^{\omega}$ sur $\overline{E_{x}} \backslash E_{x}$. 
Avec ce résultat et le théorème 2.3, on a, pour chaque classe de Wadge $\Gamma$ non auto-duale, un test pour dire si un borélien à coupes dénombrables est $\operatorname{pot}(\Gamma)$ (en effet, un tel borélien est $\operatorname{pot}\left(F_{\sigma}\right)$ ).

\section{Références.}

[D-SR] G. Debs et J. Saint Raymond, Sélections boréliennes injectives, Amer. J. Math. 111 (1989), 519-534

[GM] S. Graf and R. D. Mauldin, Measurable one-to-one selections and transition kernels, Amer. J. Math. 107 (1985), 407-425

[HKL] L. A. Harrington, A. S. Kechris et A. Louveau, A Glimm-Effros dichotomy for Borel equivalence relations, J. Amer. Math. Soc. 3 (1990), 903-928

[Ke] A. S. Kechris, Measure and category in effective descriptive set theory, Ann. of Math. Logic, 5 (1973), 337-384

[Ku] K. Kuratowski, Topology, Vol. 1, Academic Press, 1966

[Le1] D. Lecomte, Classes de Wadge potentielles et théorèmes d'uniformisation partielle, Fund. Math. 143 (1993), 231-258

[Le2] D. Lecomte, Classes de Wadge potentielles des boréliens à coupes dénombrables, C. R. Math. Acad. Sci. Paris 317, Série 1 (1993), 1045-1048

[Lo1] A. Louveau, Livre à paraître

[Lo2] A. Louveau, Ensembles analytiques et boréliens dans les espaces produit, Astérisque (S. M. F.) 78 (1980)

[Lo-SR] A. Louveau and J. Saint Raymond, Borel classes and closed games : Wadge-type and Hurewicz-type results, Trans. A. M. S. 304 (1987), 431-467

[Ma] R. D. Mauldin, One-to-one selections, marriage theorems, Amer. J. Math. 104 (1982), 823-828

[Mo] Y. N. Moschovakis, Descriptive set theory, North-Holland, 1980

[O] J. C. Oxtoby, Measure and category, Springer-Verlag, 1971

[S] J. R. Steel, Analytic sets and Borel isomorphisms, Fund. Math. 108 (1980), 83-88

[SR] J. Saint Raymond, La structure borélienne d'Effros est-elle standard ?, Fund. Math. 100 (1978), 201-210

[W] W. W. Wadge, Thesis, Berkeley (1984) 